\title{
11. Sur l'uniformisation des complémentaires analytiques et les ensembles projectifs de la seconde classe. $\left({ }^{1}\right)$
}

\author{
Par Motokiti KonDô. \\ (reçu le 9 Septembre, 1938).
}

\section{Table des Matières.}

I. Introdution Page

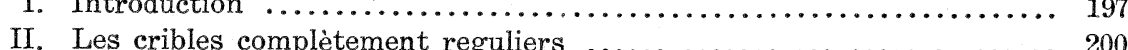

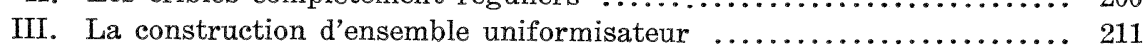

IV. Quelques applications du théorème de l'uniformisation sur des ensembles projectifs de la seconde classe $\ldots . \ldots \ldots \ldots \ldots \ldots \ldots \ldots . \ldots 223$

\section{Introduction.}

Dans sa note $\left({ }^{2}\right)$, M. N. Lusin a introduit quelques notions sur l'uniformisation des ensembles. D'après sa définition, pour un ensemble $E$ de points contenus dans le plan $X O Y$, nous dirons que $E$ est uniforme (relativement à l'axe $O X$ ), si toute paralléle à l'axe $O Y$ coupe $E$ en un point au plus. Et, nous dirons qu'un ensemble plan $E$ est uniformisable lorsqu'on a obtenu une partie uniforme $U$ de $E$ ayant la même projection sur l'axe $O X$. Cet ensemble $U$ est dit uniformisateur de $E$ et trouver tel ensemble $U$ c'est uniformiser l'ensemble donné $E$.

Sur le problème d'uniformisation des ensemble M. M. N. Lusin et W. SIERPIŃski ont démontré le

Théorème. Tout ensemble plan mesurable $(B)$ peut être uniformisé au moyen d'un complémentaire analytique. $\left({ }^{3}\right)$

Et M. W. SierpiŃsKI a posé un problème suivant:

Un ensemble $C(A)$ plan est-il toujours uniformisable (même au sens idéaliste) au moyen d'un ensemble $P C(A)$, ou même d'un ensemble projectif? ( $\left.{ }^{4}\right)$

(1) Les résultats principaux de cet note ont été présentés à l'Académie Impériale du Japon, à la séance du 12, Octobre, 1937. Voir, M. Kondô, L'uniformisation des complémentaires analytiques, Proc. of the Imp. Acad. Japan, 13 (1937), 287-291.

(2) N. Lusin, Sur le problème de M. Jacques Hadamard d'uniformisation des ensembles, Mathematica, 4 (1930), 54-66.

(3) N. Lusin, loc. cit., (2), p. 59, et W. Sifrpiński, Sur l'uniformisation des ensembles mesurables (B), Fund. Math., 16 (1930), 136-139.

(4) W. SierpińskI, loc. cit., (3), 139. 
Or, M. N. Lusin $\left({ }^{5}\right)$ a donné un complémentaire analytique plan $E$, et verifié que l'ensemble $E$ ne peut jamais être uniformisé au point de vue des réalistes. En autre part, M. P. NovikofF a démontré récemment le

Théorème. Chaque complémentaire analytique plan (situé dans un plan XOY) qui a sur chaque prallèle ḋ l'axe $O Y$ un nombre fini de points au plus peut être uniformisé au moyen d'un complémentaire analytique, $\left({ }^{6}\right)$ et recherché la classe d'ensembles donnés comme la projection des complémentaires analytiques uniformes. Or, on peut trouver sans peine une ressemblance entre la classe d'ensembles recherchés par M. P. NoviKofF et la classe d'ensembles projectifs de la seconde classe. Et, de plus, on peut savoir que le problème de l'uniformisation des complémentaires analytiques est intimement lié aux problèmes sur les ensembles projectifs de la seconde classe.

Or, M. M. N. Lusin et P. NovikofF ont donné une méthode ( $\left.{ }^{7}\right) d u$ choix effectif d'un point un complémentaire analytique arbitraire donné par un crible. En se servant de cette méthode, on peut déterminer un ensemble uniformisateur d'un complémentaire analytique plan donné. Mais, dans ce cas, le problème de l'évaluation de classe d'ensemble uniformisateur reste ouvert. Or, la méthode $\left({ }^{8}\right)$ de M. P. NovikofF se servant dans l'uniformisation de quelques complémentaires analytiques a achevé cette évaluation, et nous pouvons démontrer le

Théorème de l'uniformisation. Tout complémentaire analytique plan $E$ peut être uniformisé au moyen d'un complémentaire analytique, et de plus, si l'ensemble $E$ est donné effectivement par un crible, on peut donner aussi effectivement un uniformisateur de $E$.

Et, on peut déduire sans peine, en se servant de ce théorème, les théorèmes de M. M. N. Lusin, W. Sierpiński et P. Novikoff $\left({ }^{9}\right)$. De plus, comme j'ai dit plus haut, grâce à ce théorème, on peut résoudre divers problèmes sur les ensembles projectifs de la seconde classe, p. ex.,

Théorème. Tout ensemble projectif de la seconde classe peut être donné comme la projection uniforme d'un complémentaire analytique.

Il me parait que ce théorème est un des théorèmes fondamentaux dans la théorie des ensembles projectifs de la seconde classe.

(5) N. Lusin, loc. cit., (2), 65.

(6) P. Novikoff, Les projections des complémentaires analytiques uniformes, Recueil Mathématique, nouvelle séries, 2 (1937), 3-16.

(7) N. Lusin et P. Novikoff, Choix effectif d'un point dans un complémentaire analytique arbitraire donné par un crible, Fund. Math., 25 (1935), 559-560.

(8) P. NovikofF, loc. cit., (6), 4.

( $\left.{ }^{9}\right)$ N. Lusin, W. Sierpiński et P. Novikoff, loc. cit., (2), (3) et (6). 
Maintenant, envisageons l'exemple de M. N. Lusin qui ne peut jamais être uniformisé au point du vue des réalistes. L'exemple de M. N. Lusin est donné comme il suit:

“'Tous les polynômes $P(t)$ en $t$ à coefficients rationnels sont une infinité dénombrable: nous les supposons ranger dans un certain ordre que je ne précise pas, mais que je suppose précisé. Soit $P_{1}(t), P_{2}(t), \ldots, P_{n}(t), \ldots$ la suite considérée. D'autre part, nous prenons une courbe péanienne continue, remplissant tout un espace à une infinité dénombrable de dimensions soit $\varphi_{1}(x), \varphi_{2}(x), \ldots, \varphi_{n}(x), \ldots$ cette courbe. Cela posé, considérons la série infinie

$$
\varphi_{1}(x) P_{1}(t)+\varphi_{2}(x) P_{2}(t)+\cdots \cdots+\varphi_{n}(x) P_{n}(t)+\cdots \cdots
$$

et faisons la construction suivante. Nous prenons le plan $X O Y$ et un point $x_{0}$ fixe quelconque situé sur l'axe $O X$. A ce point fixe $x_{0}$ nous faisons correspondre la série $\left(S x_{0}\right)$ de polynômes en $t$ qu'on obtient en posant $x=x_{0}$ dans la série $(S)$ :

$$
\varphi_{1}\left(x_{0}\right) P_{1}(t)+\varphi_{2}\left(x_{0}\right) P_{2}(t)+\cdots \cdots+\varphi_{n}\left(x_{0}\right) P_{n}(t)+\cdots \cdots \cdot
$$

Enfin, nous marquons sur la droite fixe $x=x_{0}$ parallèle à l'axe $O Y$ tous les points dont les ordonnées $y$ sont des valeurs de la somme de la série de polynômes $\left(S_{x_{0}}\right)$ lorsque $t$ varie entre $-\infty$ et $+\infty$. Ce sont les points défendus. Désignons par $E$ l'ensemble plan qu'on obtient en supprimant de chaque droite $x=x_{0}$ les points marqués défendus. Je dis maintenant que l'ensemble plan $E$ ainsi défini ne peut jamais être uniformisé."'( $\left.{ }^{10}\right)$

Or, on peut uniformiser effectivement cet ensemble $E$ au moyen d'un complémentaire analytique. En effet, comme la série $(S)$ définit effectivement une fonction $F(x, t)$ de BAIRE, l'image géométrique de la fonction $y=F(x, t)$ ainsi obtenue est dans l'espace $X O Y T$ un ensemble effectivement mesurable $(B)$, et donc, la projection de cette image géométrique sur le plan $X O Y$, c'est-à-dire, l'ensemble de tous les points défendus est un ensemble effectivement analytique, d'où l'ensemble $E$ est un complémentaire effectivement analytique. Il en résulte que $E$ est d'après le théorème de l'uniformisation effectivement uniformisé au moyen d'un complémentaire analytique.

Le but de ce travail est de démontrer le théorème de l'uniformisation d'ensembles complémentaires analytiques et de donner quelques applications sur les ensembles projectifs de la seconde classe. Pour le démontrer, nous introduirons d'abord dans la chapitre II la notion des cribles complètement réguliers, et rechercherons quelques propriétés des cribles ainsi

(10) N. Lusin, lac. cit., (2), 65-66. 
définis, et dans le chapitre III nous construirons un uniformisateur d'un complémentaire analytique plan. Et enfin, dans le chapitre IV, nous appliquerons le théorème de l'uniformisation sur les ensembles projectifs de la seconde classe.

\section{Les notations utilisées dans la suite.}

$R(x), R(x, y), R(x, y, z), \ldots$ : respectivement l'ensemble de tous les nombres irrationnels $x$ tels qu'on ait $0<x<1$, de tous les paires $(x, y)$ de nombres irrationnels $x$ et $y$ tels qu'on ait $0<x<1$ et $0<y<1$, etc, et nous appellerons ces ensembles les domaines fondamentaux.

$R_{0}(x)$ : l'ensemble de tous les nombres rationnels $x$ tels qu'on ait $0 \leqq x \leqq 1$.

$R_{0}(x ; y), \quad R_{0}(x, y ; z), \ldots$ : respectivement le produit $R(x) \times R_{0}(y)$, $R(x, y) \times R_{0}(z)$, etc.

$C^{(p)}$ : soient $C$ un ensemble contenu dans un espace $X_{1} O X_{2} X_{3} \ldots X_{n}$ à $n$ dimensions, et $p$ un point de l'espace $X_{1} O X_{2} X_{3} \ldots X_{k}$ à $k$ dimensions $(k<n)$, désignons par $C^{(p)}$ l'ensemble de tous les points de $C$ dont les projections sur l'espace $X_{1} O X_{2} X_{3} \ldots X_{k}$ sont le point $p$.

$\tau(C): C$ étant un ensemble de nombres réels, si $C$ est bien ordonné suivant la grandeur de nombre réels, désignons par $\tau(C)$ le type d'ordre de $C$, et sions, posons $\tau(C)=\Omega$. De plus, si l'on $\tau(C)=\Omega$, posons $\tau(C)+1=\Omega$.

${ }^{(p)}(\ldots \ldots \ldots \ldots \ldots):$ l'ensemble de tous les points $p$ qui satisfont à la condition $(\ldots \ldots \ldots \ldots \ldots)$.

\section{Les cribles complètement reguliers.}

1. Les cribles complètement réguliers. Étant donné un ensemble analytique $E$ dans $R(x, y)$, prenons un crible $C$ dans $R_{0}(x, y ; z)$ qui défini $E$, c'est-à-dire, $E$ est l'ensemble de tous les points $p$ du plan $X O Y$, tels que l'ensemble $C^{(p)}$ ne soit pas bien ordonné suivant la diréction positive de l'axe $O Z$. Si le crible $C$ jouit des propriétés suivantes, $C$ est appelé le crible complètement régulier ;

$1^{\circ}, C$ est un ensemble d'une infinité dénombrable de rectangulaires de BAIRE situés sur des plans parallèles au plan $X O Y$,

$2^{\circ}$, quelque soit le nombre rationnel $r$, la partie de $C$-désignons par $C_{r}$-située au dessous du plan parallèle au plan XOY qui passe par le point $r$ de l'axe $O Z$ est régulier au sens de M. P. Novikoff $\left({ }^{11}\right)$, c'est-à-

11) P. Novikoff, Sur la separabilité des ensembles projectifs de seconde classe, Fund. Math., 25 (1935), 459-566. 
dire, pour chaque point $p$ du plan $X O Y, C_{r}^{(p)}$ contient une partie dense en-soi ou bien $C_{r}^{(p)}$ est bien ordonné par rapport à la direction positive de l'axe $O Z$,

$3^{\circ}$, pour tout point $p$ de $R(x, y), C^{(p)}$ contient une infinité dénombrable de points.

Lemme 1. Etant donné un ensemble analytique $E$ dans $R(x, y)$, il existe dans $R_{0}(x, y ; z)$ un crible complètement régulier qui définit l'ensemble analytique $E$.

Démonstration. Pour l'ensemble analytique $E$, on choisit un schème de SousLIN $\left\{E n_{1} n_{2} \ldots n_{k}\right\}\left(k, n_{k}=1,2, \ldots\right)$ de rectangles de BAIRE tel qu'on ait $E=\sum_{(i)} \prod_{k=1}^{\infty} E_{n_{1}} n_{2} \ldots n_{k}$. Ici, on peut supposer qu'on ait pour toute suite $\left\{n_{k}\right\}(k=1,2, \ldots)$ de nombres naturels

$$
E n_{1}=R(x, y) \quad \text { et } \quad E n_{1} n_{2} \ldots n_{k}>E n_{1} n_{2} \ldots n_{k} n_{k+1} .
$$

Prenons une fonction $\mu(n)$ de nombres naturels telle que pour tout nombre naturel $k$ il existe une infinité dénombrable de nombres naturels $n$ tels qu'on ait $k=\mu(n)$, et on définira dans $R_{0}(x, y ; z)$ l'ensemble

où

$$
\begin{aligned}
& C=\sum_{n_{1} n_{2} \ldots n_{k}} E_{\mu\left(n_{1}\right) \mu\left(n_{2}\right) \ldots \mu\left(n_{k}\right)} \times\left(z_{n_{1} n_{2} \ldots n_{k}}\right), \\
& z_{n_{1} n_{2} \ldots n_{k}}=1-\sum_{j=1}^{k} 2^{-\left(n_{1}+n_{2}+\cdots+n_{j}\right)} .
\end{aligned}
$$

On peut voir alors que $C$ est un crible complètment régulier qui définit l'ensemble analytique $E$.

Pour cela, nous allons d'abord démontrer que le crible $C_{r}$ est régulier au sens de M. P. NovikofF pour tout nombre rationnel $r$. Maintenant, supposons que pour un point $p$ du plan $X O Y$ l'ensemble $C_{r}^{(p)}$ contient une suite $\left\{z^{(k)}\right\}(k=1,2, \ldots)$ de nombres rationnels telle qu'on ait $z^{(k)}>z^{(k+1)}$ $(k=1,2, \ldots)$. On peut d'après la définition de $C$ choisir les nombres naturels $n_{i}^{(k)}\left(i=1,2, \ldots \nu_{k}, k=1,2, \ldots\right)$ tels qu'on ait

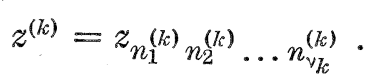

Selon la monotonité de $z^{(k)}$, nous avons $n_{1}^{(1)} \geqq n_{1}^{(2)} \geqq n_{1}^{(3)} \geqq \cdots \geqq 1$. Il existe donc les deux nombres naturels $n_{1}$ et $N_{1}$ tels qu'on ait $n_{1}^{(k)}=n_{1}$ pour $k>N_{1}$. Or, nous avons pour tout nombre naturel $k$ tel qu'on ait $k>N_{1}, n_{2}^{(k)} \geqq n_{2}^{(k+1)} \geqq n_{2}^{(k+2)} \geqq \cdots \geqq 1$. On peut donc choisir les deux nombres naturels $n_{2}$ et $N_{2}$ tels qu'on ait $n_{2}^{(k)}=n_{2}$ pour $k>N_{2}$ et $N_{2}>N_{1}$. De même, on peut déterminer de proche en proche les nombres naturels $n_{k}$ et $N_{k}(k=3,4, \ldots)$ de la manière qu'on a $n_{i}^{(k)}=n_{k}$ pour $k>N_{i}$ et $N_{i}>N_{i-1}$. Posons maintenant $\hat{z}^{(k)}=z n_{1} n_{2} \ldots n_{k}$. Nous avons alors $\quad \hat{z}^{(k)}>\hat{z}^{(k+1)}$ 
$(k=1,2, \ldots)$. Or, on peut écrire pour $k>N_{l}, z^{(k)}=z_{n_{1} n_{2} \ldots n_{l} n_{l+1}^{(k)} \ldots n_{\gamma_{k}}^{(k)},}$ d'où nous avons pour $k>N_{l}$

$$
\begin{aligned}
z^{(k)}=\hat{z}^{(l)}-2^{-\left(n_{1}+\cdots+n l\right)}( & 2^{-n_{l+1}^{(k)}}+2^{-\left(n_{l+1}^{(k)}+n_{l+1}^{(k)}\right)} \\
& \left.+\cdots+2^{-\left(n_{l+1}^{(k)}+n_{l+2}^{(k)}+\cdots+n_{v_{k}}^{(k)}\right)}\right),
\end{aligned}
$$

ce qui donne encore pour $k>N_{l}$

$$
\hat{z}^{(l)}<z^{(k)}+2^{-\left(n_{1}+n_{2}+\cdots+n_{l}\right)} \quad(l=1,2, \ldots) .
$$

Or, nous avons d'après la définition de $C_{r} \quad r>z^{(1)}>z^{(k)}$. Par suite, on peut choisir un nombre naturel $N$ de la façon qu'on a $r>\hat{z}^{(l)}$ pour $l>N$. D'alleurs, nous avons pour $k>N_{l}$

$$
p \in E_{\mu\left(n_{1}^{(k)}\right) \mu\left(n_{2}^{(k)}\right) \ldots \mu\left(n_{\nu_{k}}^{(k)}\right)}<E_{\mu\left(n_{1}\right) \mu\left(n_{2}\right) \ldots u\left(n_{l}\right)},
$$

d'où nous avons $\hat{z}^{(l)} \in C^{(p)}(l=1,2, \ldots)$ et par suite $\hat{z}^{(l)} \in C_{r}^{(p)}$ pour $l>N$. Maintenant, nous désignons par $Q$ l'ensemble de tous les nombres rationnels $z m_{1} m_{2} \ldots m_{k}$ tels qu'on ait

$$
z_{m_{1} m_{2} \ldots m_{k}} \in C_{r}^{(p)} \quad \text { et } \quad \mu\left(n_{i}\right)=\mu\left(m_{i}\right) \quad(i=1,2, \ldots k) .
$$

Puisque nous avons $\hat{z}^{(l)} \in C_{r}^{(p)}$ pour $l>N$, les nombres rationnels $\hat{z}^{(l)}$ sont contenus dans l'ensemble $Q$, ce qui donne $Q \neq 0$. Or, on peut voir que $Q$ est dense en-soi. En effet, prenons un nombre rationnel $z m_{1} m_{2} \ldots m_{k}$ dans $Q$. Il existe une infinité dénombrable de nombres naturels $m$ tels qu'on ait $\mu\left(n_{k+1}\right)=\mu(m)$; désignons par $m^{(l)}(l=1,2, \ldots)$ ces nombres naturels. Nous avons alors

et

$$
\begin{aligned}
& r>z_{m_{1} m_{2} \ldots m_{k}}>z_{m_{1} m_{2} \ldots m_{k} m^{(l)}}, \\
& \lim _{l \rightarrow \infty} z_{m_{1} m_{2} \ldots m_{k} m^{(l)}}=z_{m_{1} m_{2} \ldots m_{k}},
\end{aligned}
$$

$$
p \in E_{\mu\left(n_{1}\right) \mu\left(n_{2}\right) \ldots \mu\left(n_{k}\right) \mu\left(n_{k+1}\right)}=E_{\mu\left(m_{1}\right) \mu\left(m_{2}\right) \ldots \mu\left(m_{k}\right) \mu \cdot\left(m^{(l))}\right.} \cdot
$$

Il en résulte que les nombres rationnels $z m_{1} m_{2} \ldots m_{k} m(l)(l=1,2, \ldots)$ est contenu dans $Q$ et par suite que $z m_{1} m_{2} \ldots m_{k}$ est un nombre d'accumulation dans $Q$, ce qui donne que $Q$ est dense en-soi. Par conséquent, le crible $C_{r}$ est régulier au sens de M. P. Novikoff.

Puis, nous allons voir que $C$ est un crible qui définit l'ensemble analytique $E$. Soit $p$ un point de l'ensemble analytique $E$. Il existe alors une suite $\left\{n_{k}\right\}(k=1,2, \ldots)$ de nombres naturels telle qu'on ait 
$p \in E n_{1} n_{2} \ldots n_{k}(k=1,2, \ldots)$. Or, on peut choisir d'après la définition de $\mu(n)$ une suite $\left\{m_{k}\right\}(k=1,2, \ldots)$ de nombres naturels telle qu'on ait $\mu\left(m_{k}\right)=n_{k}(k=1,2, \ldots)$. Selon la définition du crible $C$, l'ensemble $C^{(p)}$ contient alors les nombres rationnels $z m_{1} m_{2} \ldots m_{k}(k=1,2, \ldots)$ et nous avons $z m_{1} m_{2} \ldots m_{k}>z m_{1} m_{2} \ldots m_{k} m_{k+1}$, d'où $C^{(p)}$ n'est pas bien ordonné suivant la direction positive de l'axe $O Z$.

Ensuite pour un point $p$ du plan $X O Y$, supposons que $C^{(p)}$ ne soit pas bien ordonné suivant la direction positive de l'axe $O Z$. Il existe dans l'ensemble $C^{(p)}$ une suite $\left\{z^{(k)}\right\}(k=1,2, \ldots)$ de nombres rationnels telle qu'on ait $z^{(k)}>z^{(k+1)}$. On peut alors déterminer les nombres naturels $n_{i}^{(k)}\left(i=1,2, \ldots, \nu_{k}, k=1,2, \ldots\right)$ tels qu'on ait

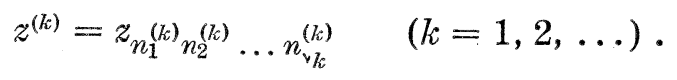

Comme nous avons fait plus haut, on peut choisir les deux suites $\left\{n_{k}\right\}$ et $\left\{N_{k}\right\}$ de nombres naturels comme il suit, $n_{i}^{(k)}=n_{i}$ pour $k>N_{i}$, et $N_{i}>N_{i-1}$. Nous avons alors pour $k>N_{l}, n_{i}^{(k)}=n_{i}(i=1,2, \ldots l)$ et par suite

$$
\begin{aligned}
E_{\mu\left(n_{1}^{(l)}\right) \mu\left(n_{2}^{(l)}\right) \ldots \mu\left(n_{\nu_{k}}^{(k)}\right)}^{(l)} & \left.E_{\mu\left(n_{1}\right) \mu\left(n_{2}\right) \ldots \mu\left(n_{l}\right) \mu\left(n_{l}(k)\right) \ldots \mu\left(n_{\nu_{k}}^{(l)}\right)}\right) \\
& <E_{\mu\left(n_{1}\right) \mu\left(n_{2}\right) \ldots \mu\left(n_{l}\right)} \cdot
\end{aligned}
$$

Or, puisque $C^{(p)}$ contient les nombres rationnels $z^{(k)}=z_{n_{1}^{(k)}} n_{2}^{(k)} \ldots n_{v_{k}}^{(k)}$,

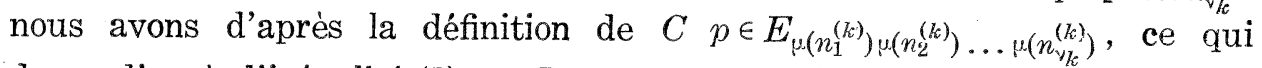
donne d'après l'inégalité (2) $\left.p \in E_{\mu \cdot\left(n_{1}\right)}\right) \cdot\left(n_{2}\right) \ldots u\left(n_{l}\right)(l=1,2, \ldots)$. Nous avons donc $p \in \prod_{l=1}^{\infty} E_{u}\left(n_{1}\right) \mu\left(n_{2}\right) \ldots \mu\left(n_{l}\right)$, d'où $p$ est un point de l'ensemble analytique $E$. Il en résulte que $C$ est un crible qui définit $E$.

Enfin, démontrons que pour tout point $p$ de $R(x, y) C^{(p)}$ contient une infinité dénombrable de points. D'après la définition des ensembles $E_{n_{1}}$, nous avons $E_{n_{1}}=R(x, y)\left(n_{1}=1,2, \ldots\right)$, d'où pour un point $p$ de $R(x, y)$ $C^{(p)}$ contient les nombres rationnels $z n_{1}\left(n_{1}=1,2, \ldots\right)$, c'est-à-dire, $C^{(p)}$ contient une infinité dénombrable de nombres rationnels.

Par suite $C$ est un crible complètement régulier qui définit l'ensemble E.

C. Q. F. D.

Remarque. Le crible $C$ est aussi régulier au sens de M. P. Novikoff. En effet, pour un point $p$ du plan $X O Y$, supposons que $C^{(p)}$ ne soit pas bien ordonné par rapport à la direction positive de l'axe $O Z$. Il existe alors un nombre rationnel $r$ tel que $C_{r}^{(p)}$ ne soit pas bien ordonné. Or, comme $C$ est complètement régulier, $C_{r}^{(p)}$ et par suite $C^{(p)}$ contient une partie dense en-soi, d'où $C$ est régulier au sens de M. P. NovikofF. 
2. La définition des ensembles $Z_{n_{1} n_{2} \ldots n_{k}}$. Définirons sur l'axe $O Z$ les intervalles.

$$
\begin{aligned}
I_{n_{1} n_{2} \ldots n_{k}}= & {\left[\sum_{1=1}^{k-1} 2^{-2\left(n_{1}+n_{2}+\cdots+n_{i}\right)+i}+2^{-2\left(n_{1}+n_{2}+\cdots+n_{k}\right)+k-1},\right.} \\
& \left.\sum_{i=1}^{k} 2^{-2\left(n_{1}+n_{2}+\cdots+n_{i}\right)+i}\right] .
\end{aligned}
$$

On peut voir alors sans peine que

$1^{\circ}$, les intervalles $I n_{1} n_{2} \ldots n_{k}$ sont disjoints deux-à-deux,

$2^{\circ}$, suivant qu'on a $z n_{1} n_{2} \ldots n_{j}>z m_{1} m_{2} \ldots m_{k}$ ou $\left\langle z m_{1} m_{2} \ldots m_{k}\right.$, nous avons $I_{n_{1} n_{2} \ldots n_{j}}>I_{m_{1} m_{2} \ldots m_{k}}$ ou $<I m_{1} m_{2} \ldots m_{k} \cdot\left({ }^{12}\right)$

Puis, prenons dans l'intervalle $I n_{1} n_{2} \ldots n_{k}$ l'ensemble $Z n_{1} n_{2} \ldots n_{k}$ de tous les nombres rationnels suivants

et

$$
w_{n_{1} n_{2} \ldots n_{k}}=\sum_{i=1}^{k_{k}-1} 2^{-\left(n_{1}+n_{2} \cdots+n_{i}\right)+i}+2^{-\left(n_{1}+\cdots+n_{k}\right)+k-1},
$$

$$
w_{n_{1} n_{2} \ldots n_{k}}+2^{-\left(n_{1} n_{2}+\cdots+n_{k}\right)+k-m} \quad(m=2,3, \ldots) .
$$

Il est alors évident que

$3^{\circ}$, l'ordre de l'ensemble $Z n_{1} n_{2} \ldots n_{k}$ est de type $\omega$ suivant la direction positive de l'axe $O Z$,

$4^{\circ}$, la relation $w n_{1} n_{2} \ldots n_{j}>w m_{1} m_{2} \ldots m_{k}$ est équivalente à la relation $z n_{1} n_{2} \ldots n_{j}>z m_{1} m_{2} \ldots m_{k}$,

$5^{\circ}$, La transformation $\varphi(t)$ qui transforme $z n_{1} n_{2} \ldots n_{j}$ en $w n_{1} n_{2} \ldots n_{j}$ établit une homéomorphe entre les ensembles $\left\{z n_{1} n_{2} \ldots n_{j}\right\}$ et $\left\{w n_{1} n_{2} \ldots n_{j}\right\}$ $\left(j, n_{j}=1,2, \ldots\right)$.

3. La modification des cribles complètement réguliers. Étant donné un schème de SousLIN $\left\{E n_{1} n_{2} \ldots n_{k}\right\}\left(k, n_{k}=1,2, \ldots\right)$ de rectangles de BAIRE qui satisfait aux condition (1) de la section 1. Considérons les deux cribles

et

$$
C=\sum_{n_{1} n_{2} \ldots n_{k}} E_{\mu \cdot\left(n_{1}\right) \mu\left(n_{2}\right) \ldots \mu\left(n_{k}\right)} \times\left(z_{n_{1} n_{2} \ldots n_{k}}\right)
$$

$$
\hat{C}=\sum_{n_{1} n_{2} \ldots n_{k}} E_{\mu\left(n_{1}\right) \mu\left(n_{2}\right) \ldots \mu\left(n_{k}\right)} \times Z_{n_{1} n_{2} \ldots n_{k}},
$$

où $\mu(n)$ est une fonction de nombres naturels telle que pour tout nombre

(12) Nous désignons par $I_{n_{1} n_{2} \ldots n_{j}}>I_{m_{1} m_{2} \ldots m_{k}}$ que pour deux points $p_{n_{1} n_{2} \ldots n_{j}}$ et $p_{m_{1} m_{2}} \ldots m_{k}$, tels qu'on ait $p_{n_{1} n_{2} \ldots n_{j}} \in I_{n_{1} n_{2}} \ldots n_{j}$ et $p_{m_{1} m_{2} \ldots m_{k}} \in I_{m_{1} m_{2} \ldots m_{k}}$, nous avons toujours $p_{n_{1} n_{2} \ldots n_{j}}>p_{m_{1} m_{2}} \ldots m_{k}$. 
naturel $k$ il existe une infinité dénombrable de nombres naturels $n$ tels qu'on ait $k=\mu(n)$.

Nous avons alors les relations suivantes entre les deux cribles $C$ et $\hat{C}$. $1^{\circ}$, Pour un point $p$ du plan $X O Y$, l'ensemble $\hat{C}^{(p)}$ contient un ensemble $Z_{n_{1} n_{2} \ldots n_{k}}$ des nombres rationnels ou bien $\hat{C}^{(p)}$ ne contient aucun nombre de l'ensemble $Z_{n_{1} n_{2} \ldots n_{k}}$, suivant que l'ensemble $C^{(p)}$ contient le nombre rationnel $z n_{1} n_{2} \ldots n_{k}$ ou non.

$2^{\circ}, \hat{C}$ est le crible complètement régulier.

En effet, étant donné un nombre rationnel $r$, supposons que pour un point $p$ du plan $X O Y$ l'ensemble $\hat{C}_{r}^{(p)}$ contient une suite $\left\{z^{(k)}\right\}(k=1,2, \ldots)$ de nombres rationnels telle qu'on ait $z^{(k)}>z^{(k+1)}$. Puisque les ensembles $Z n_{1} n_{2} \ldots n_{k}$ sont bien ordonnés suivant la direction positive de l'axe $O Z$, on peut supposer, sans perdre la généralité, que les deux nombres distincts $z^{(i)}$ et $z^{(j)}$ n'appartiennent pas au même ensemble $Z_{n_{1} n_{2} \ldots n_{k}}$. Désignons par $Z_{n_{1}^{(k)} n_{2}^{(k)} \ldots n_{y_{k}}^{(k)}}$ l'un des ensembles $Z n_{1} n_{2} \ldots n_{l}$ qui contient le nombre $z^{(k)}$.

Comme nous avons d'après l'hypothèse $z^{(k)}>z^{(k+1)}$, nous avons selon

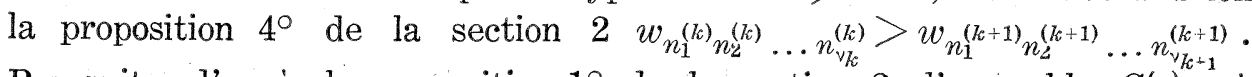
Par suite, d'après la proposition $1^{\circ}$ de la section 2 , l'ensemble $C_{r_{0}}^{(p)}$, où

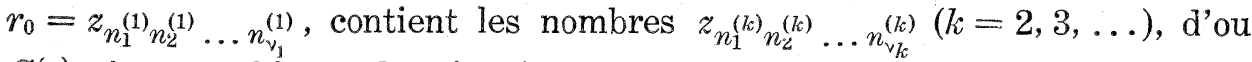
$C_{r_{0}}^{(p)}$ n'est pas bien ordonné suivant la direction positive de l'axe $O Z$. Or, puisque $C$ est un crible complètement régulier, $C_{r_{0}}^{(p)}$ contient une partie $P$ dense en-soi, d'où $\varphi(P)$ est d'après la proposition $5^{\circ}$ de la section 2 aussi partout dense en-soi. Or, l'ensemble $\varphi(P)$ appartient à l'ensemble $\hat{C}_{r}^{(p)}$, ce qui entraine que l'ensemble $\hat{C}_{r}^{(p)}$ contient une partie dense en-soi, et le crible $\hat{C}_{r}$ est donc régulier au sens de M. P. NovikofF. Il est evident que pour tout point $p$ de $R(x, y)$ l'ensemble $\hat{C}^{(p)}$ contient une infinité dénombrable de nombres rationnels. Il en résult que le crible $\hat{C}$ est complètement régulier.

$3^{\circ}$, Les deux cribles $C$ et $\hat{C}$ définissent le même ensemble analytique.

En effet, supposons que pour un point $p$ du plan $X O Y C^{(p)}$ ne soit pas bien ordonné suivant la direction positive de l'axe $O Z$. On peut alors choisir dans l'ensemble $C^{(p)}$ les nombres rationnels $z^{(k)}(k=1,2, \ldots)$ tels qu'on ait $z^{(k)}>z^{(k+1)}$. Or, d'après la définition de $\hat{C}$ les nombres rationnels $\phi\left(z^{(k)}\right)(k=1,2, \ldots)$ sont contenus dans l'ensemble $\hat{C}^{(p)}$ et $\phi\left(z^{(k)}\right)>$ $\varphi\left(z^{(k+1)}\right)$, ce qui donne que $\hat{C}^{(p)}$ n'est pas bien ordonné par rapport à la direction positive de l'axe $O Z$.

Puis, supposons que pour un point $p$ du plan $X O Y \hat{C}^{(p)}$ n'est pas bien ordonné par rapport à la direction positive de l'axe $O Z$. On peut alors choisir dans $\hat{C}^{(p)}$ une suite $\left\{z^{(k)}\right\}(k=1,2, \ldots)$ de nombres rationnels telle qu'on ait $z^{(k)}>z^{(k+1)}(k=1,2, \ldots)$. Comme nous avons fait dans la 
démonstration de la proposition $2^{\circ}$ de cette section, on peut choisir parmi l'ensemble $\left\{w n_{1} n_{2} \ldots n_{k}\right\}\left(k, n_{k}=1,2, \ldots\right)$ une suite $\left\{w^{(k)}\right\}(k=1,2, \ldots)$ de nombres rationnels dans $\hat{C}^{(p)}$ telle qu'on ait $w^{(k)}>w^{(k+1)}$. Or, d'après les définitions de $C$ et $w^{(k)}$, les nombres rationnels $\varphi^{-1}\left(w^{(k)}\right)$ sont contenus dans $C$ et $\varphi^{-1}\left(w^{(k)}\right)>\varphi^{-1}\left(w^{(k+1)}\right)$, d'où $C^{(p)}$ n'est pas bien ordonné suivant la direction positive de l'axe $O Z$. Les deux cribles $C$ et $\hat{C}$ définissent donc le même ensemble analytique.

$4^{\circ}$, Pour tout point $p$ du plan $X O Y, \hat{C}^{(p)}$ est bien ordonné de type $\alpha \omega$ ou non bien ordonné par rapport à la direction positive de l'axe $O Z$.

En effet, admettons que pour un point $p$ du plan $X O Y \hat{C}^{(p)}$ soit bien ordonné suivant la direction positive de l'axe $O Z$. Alors, $C^{(p)}$ est aussi d'après sa définition bien ordonné en même condition. Soient $a$ le type d'ordre de l'ensemble $C^{(p)}$ et $\left\{z^{(5)}\right\}(0 \leqq \xi<\alpha)$ les nombres rationnels de $C^{(p)}$ rangés de manière qu'on ait $z^{\left(\xi_{1}\right)}<z^{\left(\xi_{2}\right)}$ pour $\xi_{1}>\xi_{2}$. Or, $\hat{C}^{(p)}$ est d'après sa définition l'ensemble de tous les nombres rationnels des ensembles $Z n_{1} n_{2} \ldots n_{k}$ tels qu'on ait $\varphi\left(z^{(5)}\right) \in Z n_{1} n_{2} \ldots n_{k}$. Il en résulte que $\hat{C}^{(p)}$ est d'après la proposition $3^{\circ}$ la section 2 bien ordonné de type $\alpha \omega$.

4. La modification des cribles complètement réguliers (suite). Maintenant, $n$ étant un nombre naturel, prenons tous les intervalles $\left\{I_{k}\right\}$ $(k=1,2, \ldots)$ d'intervalles de BAIRE d'ordre $n$. Et posons dans $R_{0}(x, y ; z)$.

où

$$
\begin{gathered}
H=\sum_{k=1}^{\infty} I_{k} \times\left(\sum_{j=1}^{\infty} I_{j}\right) \times\left\{\left(r_{1}\right)+\left(r_{2}\right)+\cdots+\left(r_{k}\right)\right\}, \\
\stackrel{*}{C}=\hat{C}+H, \\
r_{k}=1+\sum_{i=1}^{k} 2^{-i} .
\end{gathered}
$$

Les deux cribles $C$ et $\stackrel{*}{C}$ définissent le même ensemble analytique. En effet, pour le voir, il suffit d'après la proposition $3^{\circ}$ de la section 3 de montrer que les deux cribles $\stackrel{*}{C}$ et $\hat{C}$ définissent le même ensemble analytique. Or, nous avons pour tout point $p$ du plan $X O Y \stackrel{*}{C}^{(p)}=\hat{C}^{(p)}+H^{(p)}$ et $H^{(p)}$ est toujours bien ordonné suivant la direction positive de l'axe $O Z$. Les cribles $\stackrel{*}{C}$ et $\hat{C}$ donc définissent le même ensemble analytique. Maintenant désignons par \& l'ensemble de tous les points $p$ de $R(x, y)$ tels que $\stackrel{*}{C^{(p)}}$ soit bien ordonné suivant la direction positive de l'axe $O Z$. Comme on sait, l'ensemble $\&$ est un complémentaire analytique, et en se servant du crible $\stackrel{*}{C}$, on peut décomposer $\mathscr{\&}$ en ses constituants $\left\{\mathscr{E}_{\alpha}\right\}(\alpha<\Omega)$ c'està-dire, étant un nombre ordinal $\alpha$ de la deuxième classe au plus, en désignant par $\varepsilon_{\alpha}$ l'ensemble de tous les points $p$ de $R(x, y)$ tels que l'ordre de $\stackrel{*}{C}^{(p)}$ 
soit de type $\alpha$ suivant la direction positive de l'axe $O Z$, nous avons $E=\sum_{\alpha<\Omega} E_{\alpha}$.

Or, on peut démontrer que pour tout point $x$ de $R(x) \oint_{\alpha}^{(x)}$ est contenu dans un intervalle de BAIRE d'ordre $n$. En effet, comme on sait, le nombre ordinal $\alpha$ peut être, d'une seule façon, écrit sous la forme $\alpha=\gamma \omega+m$ et $0 \leqq m<\omega$. Maintenant, prenons un point $p=\left(x, y_{0}\right)$ de $\mathscr{E}_{\alpha}^{(x)}$, et supposons que le point $p=\left(x, y_{0}\right)$ soit contenu dans un intervalle $I_{k_{0}}$ de BAIRE d'ordre $n$. Nous avons alors

$$
\begin{aligned}
\stackrel{\leftrightarrow}{C}^{(p)}=\hat{C}^{(p)}+H^{(p)} & =\hat{C}^{(p)}+\sum_{k=1}^{\infty} I_{k}^{\left(y_{0}\right)} \times(x) \times\left\{\left(r_{1}\right)+\left(r_{2}\right)+\cdots+\left(r_{k}\right)\right\} \\
& =\hat{C}^{(p)}+\left(r_{1}\right)+\left(r_{2}\right)+\cdots+\left(r_{k_{0}}\right) .
\end{aligned}
$$

Or, d'après la définition du crible $\hat{C}$ le type d'ordre de $\hat{C}^{(p)}$ est de deuxième espéce, et donc le type d'ordre de $\hat{C}^{(p)}$ peut être écrit sous la forme $\lambda \omega$. Par conséquent, le type d'ordre de $\stackrel{C}{C}^{(p)}$ est de la forme $\lambda \omega+k_{0}$. Or, en autre part, le type d'ordre de $\stackrel{*}{C}^{(p)}$ est $\alpha=\gamma \omega+m$, d'où nous avons $\gamma \omega+m=\lambda \omega+k_{0}$, ce qui donne $k_{0}=m$. Il en résulte que le point $p$ est contenu dans l'intervalle $I_{m}$ de BAIRE d'ordre $n$. Par conséquent, $\mathscr{E}_{\alpha}^{(x)}$ est contenu dans l'intervalle $I_{m}$ de BAIRE d'ordre $n$.

Donc, en posant la définition suivante, on peut résumer les résultats ainsi obtenus comme il suit.

Définition. Soient $C$ un crible complètement régulier défini dans $R_{0}(x, y ; z)$ et $\&$ l'ensemble de tous les points $p$ de $R(x, y)$ tels que $C^{(p)}$ soit bien ordonné suivant la direction positive de l'axe $O Z$. En se servant du crible $C$, on peut décomposer $\&$ en ses constituantes $\mathscr{E}_{\alpha}(0 \leqq \alpha<\Omega)$, où $\mathscr{E}_{\alpha}$ est l'ensemble de tous les points $p$ de $\&$ tels que l'ordre de $C^{(p)}$ soit de type $\alpha$. Si l'ensemble $\mathscr{E}_{\alpha}^{(x)}$ est toujours contenu dans un intervalle de BAIRE d'ordre $n$, quels que soient les points $x$ de $R(x)$ et le nombre ordinal $\alpha$ de la deuxième classe au plus, nous appellons $C$ un crible complètement régulier de classe $n$.

Lemme 2. Soient $n$ un nombre naturel et $E$ un ensemble analytique contenu dans $R(x, y)$. On peut choisir un crible complètement régulier de classe $n$ qui définit l'ensemble analytique $E$.

5. La décomposition des cribles complètement réguliers (de classe $n$ ). Étant donné un crible $C$ complètement régulier, rangeons tous les rectangles du crible $C$ en une suite infine $\left\{J_{k}\right\}(k=1,2, \ldots)$. En désignant par $\lambda_{k}$ l'ordre du rectangle $J_{k}$ de BAIRE, posons

$$
\lambda_{k}^{*}=\max \left(\lambda_{1}, \lambda_{2}, \ldots, \lambda_{k}\right)+k \quad(k=1,2, \ldots) .
$$


Nous avons alors $\lambda_{k}<\lambda_{k}^{*}<\lambda_{k+1}^{*}$. Maintenant nous décomposerons le rectangle $J_{k}$ en les rectangles partiels de BAIRE d'ordre $\lambda_{k}^{*}$, et désignons par $J_{k n}$ $(n=1,2, \ldots)$ ces rectangles partiels de BAIRE d'ordre $\lambda_{k}^{*}$. Nous avons alors $C=\sum_{k=1}^{\infty} \sum_{n=1}^{\infty} J_{k n}$ et les projections sur le plan $X O Y$ des deux rectangles distincts $J_{k_{i} n_{i}}(i=1,2)$ de la famille $\left\{J_{k n}\right\}(k, n=1,2, \ldots)$ sont distincts deux-à-deux. En effet, si l'on a $k_{1}=k_{2}$, nous avons d'après la définition de $J_{k n}$ les projections de $J_{k_{1} n_{1}}$ et $J_{k_{2} n_{2}}$ sont disjoints deux-à-deux. Si l'on $k_{1} \neq k_{2}$, on peut supposer, sans perdre la généralité, qu'on ait $k_{1}<k_{2}$. Nous avons alors $\lambda_{k_{1}}^{*}>\lambda_{k_{2}}^{*}$ c'est-à-dire, l'ordre $\lambda_{k_{1}}^{*}$ de $J_{k_{1} n_{1}}$ est inférieur à l'ordre $\lambda_{k_{2}}$ de $J_{k_{2} n_{2}}$. Les projections de ces rectangles donc distincts deuxà-deux.

Maintenant, en se servant des rectangles $J_{k n}$, nous allons définir un schème de SousLIN $\left\{H n_{1} n_{2} \ldots n_{k}\right\}\left(k, n_{k}=1,2, \ldots\right)$ de rectangles de BAIRE comme il suit. Les projections (sur le plan $X O Y$ ) de deux rectangles de la famille $\left\{J_{k n}\right\}$ sont disjoints deux-à-deux, ou bien l'un est contenu dans l'autre. Par suite, il existe dans la famille $\left\{J_{k n}\right\}$ au moins et par suite une infinité dénombrable de rectangles $J_{k n}$ tels que la projection sur le plan $X O Y$ de $J_{k n}$ n'appartient à aucune projection d'autre rectangle de la famille $\left\{J_{k n}\right\}$. Choisissons parmi la famille $\left\{J_{k n}\right\}$ tous les tels rectangles et désignons par $H n_{1}\left(n_{1}=1,2, \ldots\right)$ ces rectangles ainsi obtenus. Puis, prenons dans la famille $\left\{J_{k n}\right\}$ tout rectangle $J_{k n}$ dont la projection sur le plan $X O Y$ n'appartient qu'à la projection de $H_{n_{1}}$ sur le même plan et désignons par $H n_{1} n_{2}\left(n_{2}=1,2, \ldots\right)$ ces rectangles ainsi obtenus. D'une manière générale, les rectangles $H n_{1} n_{2} \ldots n_{i}(i=1,2, \ldots k)$ étant définis, nous prenons dans la famille $\left\{J_{k n}\right\}$ tout rectangle $J_{k_{n}}$ dont la projection sur le plan $X O Y$ n'appartient qu'aux projections de $H n_{1} n_{2} \ldots n_{i}(i=1,2, \ldots k)$ sur le même plan et désignons par $H_{n_{1} n_{2} \ldots n_{k} n_{k+1}}\left(n_{k+1}=1,2, \ldots\right)$ ces rectangles ainsi obtenus.

Quand on désigne par $G n_{1} n_{2} \ldots n_{k}$ la projection de $H n_{1} n_{2} \ldots n_{k}$ sur le plan $X O Y$, nous pouvons démontrer les propositions suivantes.

$1^{\circ}$. Quel que soit le point $p$ de $R(x, y)$, il existe une suite $\left\{n_{k}\right\}$ $(k=1,2, \ldots)$ de nombres naturels telle qu'on ait $(p)=\prod_{k=1}^{\infty} G n_{1} n_{2} \ldots n_{k}$.

En effet, étant donné un point $p$ de $R(x, y)$, considérons tous les rectangles $J_{k n}$ tels que la projection de $J_{k n}$ sur le plan $X O Y$ contienne le point $p$. D'après la définition de $C$, il existe dans la famille $\left\{J_{k n}\right\}$ une infinité dénombrable de tels rectangles. On peut les ranger d'après la définition de $J_{k n}$ une suite infine $\left\{J_{k_{i} n_{i}}\right\}(i=1,2, \ldots)$ de la manière que $k_{i}<k_{i+1}$ $(i=1,2, \ldots)$. Comme le rectangle $J_{k_{i} n_{i}}$ est d'ordre $\lambda_{k_{i}}^{*}$, nous avons ProjxoY $J_{k_{i} n_{i}}>\operatorname{Proj}_{X O Y} J_{k_{i+1} n_{i+1}}$ et $\prod_{i=1}^{\infty} \operatorname{Proj}_{X O Y} J_{k_{i} n_{i}}=(p)$. Or, 11 existe une suite $\left\{m_{k}\right\}(k=1,2, \ldots)$ de nombres naturels telle qu'on ait $J_{k_{i} n_{\boldsymbol{i}}}=H m_{1} m_{2} \ldots m_{i}$. 
En effet, puisque tout rectangle de la famille $\left\{J_{k n}\right\}$, tel qu'on ait $p \in \operatorname{Proj}_{X O Y}$ $J_{k n}$, est contenu dans la suite $\left\{J_{k_{i} n_{i}}\right\}(i=1,2, \ldots)$ et qu'on a Proj $j_{X O Y}$ $J_{k_{1} n_{1}}>\operatorname{Proj}_{X O Y} J_{k_{i} n_{i}}$, il existe donc un nombre naturel $m_{1}$, tel qu'on ait $J_{k_{1} n_{1}}=H m_{1}$. Puis, tout rectangle $J_{k n}$ tel qu'on ait $p \in$ Proj $_{X O Y} J_{k n}$ et qu'on ait ProjXOY $J_{k n}<\operatorname{Proj}_{X O Y} H m_{1}$ est un des rectangles $J_{k_{i} n_{i}}$ et $\operatorname{Proj}_{X O Y} J_{k_{i} n_{i}}$ $<\operatorname{Proj}_{X O Y} J_{k_{2} n_{2}}(i=2,3, \ldots)$, il existe donc un nombre naturel $m_{2}$ tel qu'on ait $J_{k_{2} n_{2}}=H m_{1} m_{2}$. De même, on peut démontrer de proche en proche qu'il existe les nombres naturels $\left\{m_{k}\right\}(k=3,4, \ldots)$, tel qu'on ait $J_{k_{i} n_{i}}$ $=H m_{1} m_{2} \ldots m_{i}$. Il existe donc une suite $\left\{m_{k}\right\}(k=1,2, \ldots)$ de nombres naturels telle qu'on ait $(p)=\prod_{i=1}^{\infty} G m_{1} m_{2} \ldots m_{i}$.

$2^{\circ}$. Pour deux suites distinctes $\left\{n_{k}^{(i)}\right\}(i=1,2 ; k=1,2, \ldots) \mathrm{de}$ nombres naturels, nous avons $\prod_{k=1}^{\infty} G_{n_{1}^{(1)}} n_{2}^{(1)} \ldots n_{k}^{(1)} \prod_{k=1}^{\infty} G_{n_{1}^{(2)}} n_{c}^{(2)} \ldots n_{k}^{(2)}=0$.

En effet, soient $\left\{n_{k}^{(2)}\right\}(i=1,2 ; k=1,2, \ldots)$ les deux suites distinctes de nombres naturels. Il existe alors un nombre naturel $j$ tel qu'on ait

$$
n_{1}^{(1)}=n_{1}^{(2)}, n_{1}^{(2)}=n_{2}^{(2)}, \ldots \ldots, n_{j}^{(1)}=n_{j}^{(2)}, n_{j+1}^{(1)} \neq n_{j+1}^{(2)} .
$$

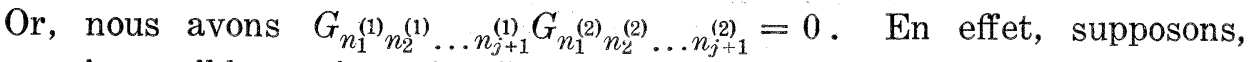

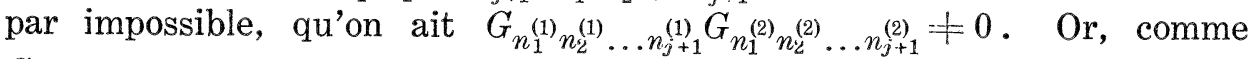
$G_{n_{1}^{(1)} n_{1}^{(1)} \ldots n_{j+1}^{(1)}}$ et $G_{n_{1}^{(2)} n_{2}^{(2)} \ldots n_{j+1}^{(2)}}$ sont les rectangles de BAIRE, on peut sup-

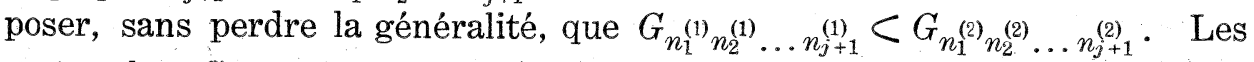
rectangles $G_{n_{1}^{(1)} n_{2}^{(1)} \ldots n_{i}^{(1)}}(i=1,2, \ldots j)$ et $G_{n_{1}^{(2)} n_{2}^{(2)} \ldots n_{j+1}^{(2)}}$ sont distincts deux-à-deux et contiennent $G_{n_{1}^{(1)} n_{2}^{(1)} \ldots n_{j+1}^{(1)}}$ respectivement, ce qui donne

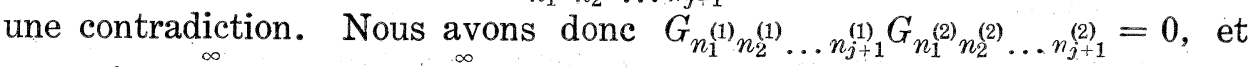

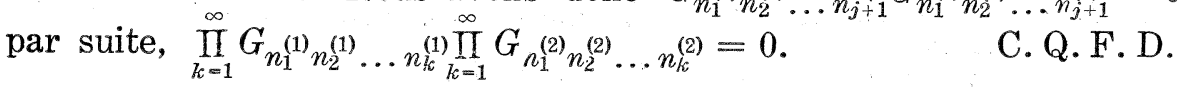

$3^{\circ}$. Pour tout suite $\left\{n_{k}\right\}(k=1,2, \ldots)$ de nombres naturels, la partie commune $\prod_{k=1}^{\infty} G n_{1} n_{2} \ldots n_{k}$ est précisément un ensemble d'un point.

En effet, étant donné une suite $\left\{m_{k}\right\}(k=1,2, \ldots)$ de nombres naturels, considérons les ensembles $H m_{1} m_{2} \ldots m_{k}$. Il existe d'après la définition de $H m_{1} m_{2} \ldots m_{i}$ une suite $\left\{\left(k_{i}, n_{i}\right)\right\}(i=1,2, \ldots)$ de paires de nombres naturels, telle qu'on ait $H m_{1} m_{2} \ldots m_{i}=J_{k_{i} n_{i}}$. Puisque les ensembles Proj ${ }_{X O Y} J_{k_{i} n_{i}}$ sont distincts deux-à-deux et que nous avons ProjXoY $J_{k_{i} n_{i}}>\operatorname{Proj}_{X O Y} J_{k_{i+1} n_{i+1}}$, nous avons $k_{i}<k_{i+1}(i=1,2, \ldots)$, et par suite, le rectangle $\operatorname{Proj}_{X O Y} J_{k_{i} n_{i}}=G m_{1} m_{2} \ldots m_{i}$ est de l'ordre $\geq i$. Donc, le produit $\prod_{i=1}^{\infty} G m_{1} m_{2} \ldots m_{i}$ ne contient qu'un point. C. Q. F. D.

$4^{\circ}$. La famille $\left\{J_{k n}\right\}(k, n=1,2, \ldots)$ de rectangles de BAIRE concide précisément avec la famille $\left\{H n_{1} n_{2} \ldots n_{k}\right\}\left(k, n_{k}=1,2, \ldots\right)$. 
En effet, comme tout ensemble de la famille $\left\{H n_{1} n_{2} \ldots n_{k}\right\}$ appartient à la famille $\left\{J_{k n}\right\}(k, n=1,2, \ldots)$, il suffit de montrer que tout ensemble de la famille $\left\{J_{k n}\right\}$ appartient à la famille $\left\{H n_{1} n_{2} \ldots n_{k}\right\}$. Considérons un ensemble $J_{k_{0} n_{0}}$ de la famille $\left\{J_{k_{n}}\right\}$. Prenons maintenant tout rectangle $J_{k n}$ tel qu'on ait Proj $\operatorname{PoY}_{X O Y} J_{k_{0} n_{0}}<\operatorname{Proj}_{X O Y} J_{k_{n}}$. Il existe d'après la définition de $J_{k n}$ un nombre fini de tels ensembles, et on peut ranger une suite finie

$$
J_{k_{1} n_{1}}, J_{k_{2} n_{2}}, \cdots, J_{k_{j} n_{j}}=J_{k_{0} n_{0}},
$$

de la manière que $k_{i}<k_{i+1}(i=1,2, \ldots j-1)$. De même que nous avons fait dans la démonstration de la proposition $1^{\circ}$ de cette section, on peut démontrer qu'il existe un nombre fini de nombres naturels $m_{k}(k=1,2, \ldots)$, tels qu'on ait $J_{k_{i} n_{i}}=H m_{1} m_{2} \ldots m_{i}$. Donc, $J_{k_{0} n_{0}}$ appartient à la famille $\left\{H_{1} n_{2} \ldots n_{k}\right\}$.

C. Q.F.D.

Grâce à cette proposition, on peut écrire le crible $C$ comme la somme $C=\sum_{n_{1} n_{2} \ldots n_{k}} H n_{1} n_{2} \ldots n_{k}$ de rectangles $H n_{1} n_{2} \ldots n_{k}$ de BAIRE, ou en désignant par $r_{n_{1} n_{2} \ldots n_{k}}$ la projection des rectangles $H n_{1} n_{2} \ldots n_{k}$ sur l'axe $O Z$, nous avons

$$
C=\sum_{n_{1} n_{2} \ldots n_{l}} G_{n_{1} n_{2} \ldots n_{k}} \times\left(r_{n_{1} n_{2} \ldots n_{k}}\right) .
$$

Nous avons done le

Lemma 3. Soient $E$ un ensemble analytique centenu dan $R(x, y)$ et $n$ un nombre naturel. "On peut définir un schème de SousLIN $\left\{G n_{1} n_{2} \ldots n_{k}\right\}$ $\left(k, n_{k}=1,2, \ldots\right)$ de rectangles de BAIRE et un système $\left\{r n_{1} n_{2} \ldots n_{k}\right\}$ $\left(k, n_{k}=1,2, \ldots\right)$ de nombres rationnels comme il suit,

$(\alpha), C=\sum_{n_{1} n_{2} \ldots n_{k}} G n_{1} n_{2} \ldots n_{k} \times\left(r n_{1} n_{2} \ldots n_{k}\right)$ est un crible complètement régulier de classe $n$ qui définit l'ensemble analytique $E$,

$(\beta)$, quel que soit le point $p$ de $R(x, y)$, il existe une suite $\left\{n_{k}\right\}$ $(k=1,2, \ldots)$ de nombres naturels telle qu'on ait $(p)=\prod_{k=1}^{\infty} G n_{1} n_{2} \ldots n_{k}$,

$(\gamma)$, pour toute suite $\left\{n_{k}\right\}(k=1,2, \ldots)$ de nombres naturels, la partie commune $\prod_{k=1}^{\infty} G n_{1} n_{2} \ldots n_{k}$ est non-vide et ne contient qu'un point.

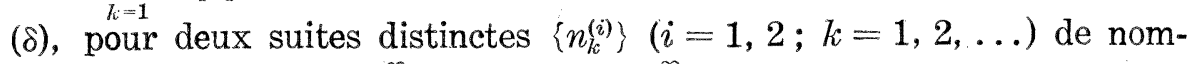

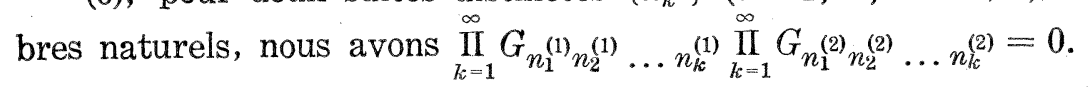

6. L'effectivité des ensembles analytiques. Pour simplifier la description, nous ne considérons dans la suite que les ensembles analytiques contenus dans un domaine fondamental. Soit $E$ un ensemble analytique contenu dans un domaine fondamental $R$ de quelque dimensions. Si l'on peut nommer un schème de SousLin $\left\{E n_{1} n_{2} \ldots n_{k}\right\}\left(k, n_{k}=1,2, \ldots\right)$ de 
parallélépipédes de BAIRE tel qu'on ait $E=\sum_{(v)} \prod_{k=1}^{\infty} E n_{1} n_{2} \ldots n_{k}$. Nous appellons $E$ un ensemble effectivement analytique et $R-E$ un complémentaire effectivement analytique. Nous avons alors sans peine les suivants.

$1^{\circ}$. Soit $\left\{E_{n}\right\}(n=1,2, \ldots)$ une suite infinie d'ensembles effectivement analytiques. Alors, la somme $\sum_{n=1}^{\infty} E_{n}$ et la partie commune $\prod_{n=1}^{\infty} E_{n}$ de ces ensembles sont aussi effectivement analytiques en même temps.

$2^{\circ}$. $E$ étant un ensemble effectivement analytique dans un domaine fondamental $R\left(x_{1}, x_{2}, \ldots, x_{n}\right)$. La projection de $E$ sur le plan $x_{n}=0$ est aussi effectivement analytique.

$3^{\circ}$. Pour qu'un ensemble analytique $E$ contenu dans un domaine fondamental soit effectivement analytique, il faut et il suffit qu'il existe une suite infinie $G_{n}(n=1,2, \ldots)$ de parallélépipèdes et une suit infinie $\left\{r_{n}\right\}(n=1,2, \ldots)$ de nombres rationnels telles que le crible $C=\sum_{n=1}^{\infty} G_{n} \times\left(r_{n}\right)$ définit l'ensemble analytique $E$.

De plus, en se servant des propositions $1^{\circ}-3^{\circ}$ de cette section, on peut démontrer, suivant la marche des démonstrations des lemmes 1-3, que $E$ étant un ensemble effectivement analytique, on peut donner effectivement un crible complètement régulier qui définit $E$.

Grâce à ces propositions en se servant de la méthode $\left({ }^{13}\right)$ de M. N. Lusin sur les cribles, nous avons le

Lemme 4. Soient $C_{1}$ et $C_{2}$ les deux cribles réguliers au sens de M. P. Novikoff contenus dans $R_{0}\left(x_{1}, x_{2}, \ldots x_{n} ; y\right)$ qui consiste en une infinité dénombrable de parallélépipèdes de BAIRE situés sur les plans parallélés au plan $y=0$. Alors les deux ensembles de tous les points $p$ de $R\left(x_{1}, x_{2}, \ldots x_{n}\right)$ tels qu'on ait $\tau\left(C_{1}^{(p)}\right) \geq \tau\left(C_{2}^{(p)}\right)$ et $\tau\left(C_{1}^{(p)}\right)=\tau\left(C_{2}^{(p)}\right)$ sont effectivement analytiques en même temps.

\section{La construction d'ensemble uniformisateur.}

7. Le théorème de l'uniformisation. Nous allons démontrer dans ce paragraphe le théorème de l'uniformisation des complémentaires analytiques :

Théorème de l'uniformisation. Tout complémentaire analytique plan $E$ peut être uuiformisé au moyen d'un complémentaire analytique, et de plus, si l'ensemble $E$ est donné effectivement par un crible, on peut donner. aussi effectivement un uniformisateur de $E$.

Pour le démontrer, nour allons donner quelques modifications du théorème dans la suite. Comme tout ensemble analytique peut être défini

(13) N. Lusin, Leçons sur les ensembles analytiques, Paris, (1930), 213. 
par un crible, pour le voir, il suffit de démontrer le cas où l'ensemble $E$ est donné effectivement par un crible. Puisque l'ensemble de tous les nombres rationnels est effectivement dénombrable, on peut ranger effectivement ces nombres rationnels en une suité infinie $\left\{r_{k}\right\}(k=1,2, \ldots)$. Nous avons alors

$$
E=\sum_{k=1}^{\infty}\left\{I_{k} \times(O Y)\right\}\left\{E-\sum_{n=1}^{\infty}\left(\left(r_{n}\right) \times(O Y)\right) E\right\}+\sum_{n=1}^{\infty}\left(\left(r_{n}\right) \times(O Y)\right) E
$$

où $I_{k}(k=0, \pm 1, \pm 2, \ldots)$ désigne l'intervalle ouvert $(k, k+1)$. Or, grâce à la méthode donné par Mi. M. N. LuSiN et P. NovikoFF( $\left.{ }^{14}\right)$, on peut choisir effectivement un point parmi chaque ensemble $\left(\left(r_{n}\right) \times(O Y)\right) E$ qui n'est pas vide. Par suite, pour démontrer le théorème de l'uniformisation il suffit de trouver effectivement un uniformisateur de $\left\{I_{k} \times(O Y)\right\}$ $\left\{E-\sum_{n=1}^{\infty}\left(\left(r_{n}\right) \times(O Y)\right) E\right\}$. Puisque $\left\{I_{k} \times(O Y)\right\}\left\{E-\sum_{n=1}^{\infty}\left(\left(r_{n}\right) \times(O Y)\right) E\right\}$ est un complémentaire effectivement analytique contenu dans $I_{k}^{*} \times(O Y)$, où $I_{k}^{*}$ est l'ensemble de tous les nombres irrationnels contenus dans $I_{k}$ et donc homéomorphe à $R(x)$, nous pouvons donc supposer, sans perdre la généralité, que le complémentaire analytique donné $E$ soit contenu dans l'ensemble $R(x) \times(O Y)$. Or, on peut transformer effectivement l'axe $O Y$ en un ensemble fermé $N$ relativement à $R(y)$ par une transformation-désignons par $\phi(y)$-qui donné une homéomorphie généralisée (0.2) entre l'axe $O Y$ et $N$. Selon la transformation

$$
\sigma(p): \quad x^{\prime}=x, \quad y^{\prime}=\phi(y),
$$

on peut établir une homéomorphe généralisée $(0,2)$ entre $E$ et $\sigma(E)$. Or, tout complémentaire effectivement analytique est transformé par cette homéomorphe généralisée en un complémentaire effectivement analytique et par suite, en particulier $\sigma(E)$ est un complémentaire effectivement analytique, et nous avons $\sigma\left(E^{(x)}\right)=(\sigma(E))^{(x)}$ pour tout point $x$ de l'axe $O X$. Donc, si l'ensemble $U$ est un uniformisateur de $\sigma(E)$, l'ensemble $\sigma^{-1}(U)$ est un uniformisateur de $E$. Et, si l'ensemble $U$ est un complémentaire effectivement analytique, $\sigma^{-1}(U)$ est aussi un complémentaire effectivement analytique. Par conséquent, pour démontrer le théorème de l'uniformisation, il suffit de démontrer que $\sigma^{-1}(E)$ peut être uniformisé au moyen d'un complémentaire effectivement analytique. Or, puisque $\sigma^{-1}(E)$ est un sousensemble de $R(x) \times R(y)=R(x, y)$, on peut supposer, sans perdre la généralité, que le complémentaire analytique donné $E$ est contenu dans $R(x, y)$.

(14) N. Lusin et P. Novikoff, loc. cit., (7), 559-560. 
8. La construction préliminaire. $E$ étant un complémentaire effectivement analytique contenu dans $R(x, y)$, on peut définir effectivement d'après le lemme 3 un schème de Souscin $\left\{G n_{1} n_{2} \ldots n_{k}\right\}\left(k, n_{k}=1,2, \ldots\right)$ de rectangles de BAIRE et un système $\left\{r n_{1} n_{2} \ldots n_{k}\right\}\left(k, n_{k}=1,2, \ldots\right)$ des nombres rationnels tels que l'ensemble

$$
C=\sum_{n_{1} n_{2} \ldots n_{k}} G_{n_{1} n_{2} \ldots n_{k}} \times\left(r_{n_{1} n_{2} \ldots n_{k}}\right)
$$

soit un crible complètement regulier qui définit l'ensemble effectivement analytique $C E=R(x, y)-E$, et tels que les rectangles de BAIRE $G n_{1} n_{2} \ldots n_{k}$ $\left(k, n_{k}=1,2, \ldots\right)$ satisfassent aux conditions $(\beta),(\gamma)$ et $(\delta)$ du lemme 3. Maintenant, désignons par $C n_{1} n_{2} \ldots n_{k}$ la partie de $C$ située rigoureusement au dessous du rectangle $G n_{1} n_{2} \ldots n_{k} \times\left(r n_{1} n_{2} \ldots n_{k}\right)$. D'après la définition du crible $C, C n_{1} n_{2} \ldots n_{k}\left(k, n_{k}=1,2, \ldots\right)$ sont les cribles réguliers au sens de M. P. Novikoff.

Maintenant, considérons dans l'espace XOYTZ à quatre dimensions une transformation qui transformè l'espace $X O Y Z$ en l'espace $X O T Z$ de la manière suivante:

$$
x^{\prime}=x, \quad y^{\prime}=0, \quad z^{\prime}=z, \quad t^{\prime}=y .
$$

Grâce à cette transformation, les cribles $C$ et $C n_{1} n_{2} \ldots n_{k}$ de l'espace $X O Y Z$ sont transformés en les cribles de l'espace $X O T Z$, désignons par $\stackrel{*}{C}$ et $\stackrel{*}{C} n_{1} n_{2} \ldots n_{k}$ ces cribles ainsi obtenus. De plus, désignons par $\stackrel{*}{G} n_{1} n_{2} \ldots n_{k}$ l'image du rectangle $G n_{1} n_{2} \ldots n_{k}$ par une transformation: $x^{\prime}=x$ et $t^{\prime}=y$, qui transforme l'espace $X O Y$ en l'espace $X O T$.

Etant donnée une suite $\left\{n_{k}\right\}(k=1,2, \ldots)$ de nombres naturels, posons pour le moment dans l'espace $X O Y T Z$

$$
\begin{aligned}
& \stackrel{(1)}{\Gamma_{0}}=C \times R(t), \quad \stackrel{(1)}{\Gamma_{k}}=C_{n_{1} n_{2} \ldots n_{k}} \times R(t), \\
& \stackrel{(2)}{\Gamma_{0}}=\stackrel{*}{C} \times R(y), \quad \stackrel{(2)}{\Gamma}=\stackrel{*}{C}_{n_{1} n_{2} \ldots n_{k}} \times R(y), \quad(k=1,2, \ldots)
\end{aligned}
$$

et encore pour tout point $p$ de $R(x, y, t)$

$$
\stackrel{(k)}{\nu_{i}}(p)=\tau\left(\Gamma_{i}^{(k)}\right) \quad(k=1,2 ; i=0,1,2, \ldots) .
$$

Et enfin, nous définissons les ensembles $M$ et $M n_{1} n_{2} \ldots n_{k}\left(k, n_{k}=1,2, \ldots\right)$ comme il suit,

$$
\begin{aligned}
& M=\stackrel{(p)}{E}\left(\stackrel{1)}{\nu_{0}}(p) \geq \stackrel{(2)}{\nu_{0}}(p)+1\right), \\
& M_{n_{1} n_{2} \ldots n_{k}}=\stackrel{(p)}{E}\left(\stackrel{(1)}{\nu_{0}}(p) \geqq \stackrel{(2)}{\nu_{0}}(p)+1\right) \\
& \left.\left.+\sum_{j=1}^{k} \stackrel{(p)}{E}\left(\stackrel{(1)}{\nu}_{0}(p)=\stackrel{(2)}{\nu_{0}}(p)\right) \stackrel{(p)}{E} \stackrel{(1)}{\left(\nu_{1}\right.}(p)=\stackrel{(2)}{\nu}_{1}(p)\right) \times \cdots \times \stackrel{(p)}{E}^{\left(\nu_{\nu}\right)}(p)=\stackrel{(2)}{\nu}_{j-1}(p)\right) \\
& \times \stackrel{(p)}{E}\left(\nu_{j}^{(1)}(p) \geqq \stackrel{(2)}{\nu_{j}}(p)+1\right) D_{j} \quad(k=1,2, \ldots) .
\end{aligned}
$$


où $D_{j}$ est l'ensemble de tous les points $(\bar{x}, \bar{y}, \bar{t})$ de $R(x, y, t)$ tels que les points $(\bar{x}, \bar{y})$ et $(\bar{x}, \bar{t})$ appartiennent à $G n_{1} n_{2} \ldots n_{j}$ et $\stackrel{*}{G} n_{1} n_{2} \ldots n_{j}$ respective-

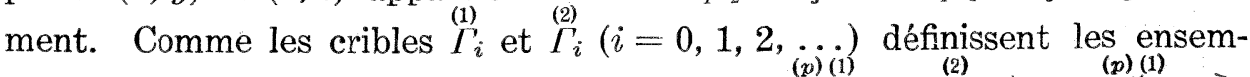
bles effectivement analytiques, les ensembles $\stackrel{(p)}{E}\left(\mathcal{\nu}_{i}(p)=\stackrel{(2)}{\nu_{i}}(p)\right)$ et $\stackrel{(p)(1)}{E}\left(\nu_{i}(p) \geqq\right.$ $\left.\nu_{i}^{(2)}(p)+1\right)$ sont d'après la lemme 4 aussi effectivement analytiques, d'où les ensembles analytiques $M$ et $M_{n_{1} n_{2} \ldots n_{k}}$ sont définis aussi effectivement.

Enfin, nous poserons

$$
\begin{aligned}
& Q=R(x, y)-\operatorname{Proj}_{X O Y} M, \\
& Q_{n_{1} n_{2} \ldots n_{k}}=G n_{1} n_{2} \ldots n_{k}-\operatorname{Proj}_{X O Y} M_{n_{1} n_{2}} \ldots n_{k} .
\end{aligned}
$$

On voit alors sans peine que les ensembles $Q$ et $Q n_{1} n_{2} \ldots n_{k}$ sont les complémentaires effectivement analytiques. Nous appellons $Q$ l'ensemble des points d'index minimum par rapport à $C$.

9. L'interprétation géométrique des ensembles $Q \boldsymbol{n}_{1} \boldsymbol{n}_{2} \ldots \boldsymbol{n}_{k}$. Pour chaque point $x$ de la projection de $E$ sur l'axe $O X$, nous désignons par $\alpha(x)$ le type minimum des types $\tau\left(C^{(x, y)}\right)$ d'ordres de $C^{(x, y)}$, lorsque $y$ pracourt $R(y)$. Comme $x$ est un point de la projection de $E$, nous avons $\alpha(x)<\Omega$. Puis, désignons par $\hat{Q}^{(x)}$ l'ensemble de tous les points $(x, y)$ de $R(x, y)$ tels qu'on ait $\tau\left(C^{(x, y)}\right)=\alpha(x)$ et par $\hat{Q}$ la somme de tous les ensembles $\hat{Q}^{(x)}$ lorsque $x$ parcourt le projection de $E$ sur l'axe $O X$. Etant donné un point $x$ de la projection de $\hat{Q} G_{1}$ sur l'axe $O X$, désignons par $a_{n_{1}}(x)$ le type minimum des types $\tau\left(C_{n_{1}}^{(x, y)}\right)$ d'ordres de $C_{n_{1}}^{(x, y)}$ lorsque $y$ parcourt $\hat{Q}^{(x)} G n_{1}$; on voit alors sans peine qu'on a $\alpha_{n_{1}}(x)<\Omega$. De plus, désignons $\operatorname{par} \dot{Q}_{n_{1}}^{(x)}$ l'ensemble de tous les points $(x, y) \in \hat{Q}^{(x)} G n_{1}$ tels qu'on ait $\tau\left(C_{n_{1}}^{(x, y)}\right)$ $=\alpha_{n_{1}}(x)$ et par $\hat{Q}_{n_{1}}$ la somme de tous les ensembles $\hat{Q}_{n_{1}}^{(x)}$, lorsque $x$ parcourt la projection de $\hat{Q} G n_{1}$ sur l'axe $O X$. D'une manière générale, $\alpha_{n_{1} n_{2} \ldots n_{j}}(x)$ et $\hat{Q}_{n_{1} n_{2} \ldots n_{j}}(j=1,2, \ldots k)$ étant définis, pour un point $x$ de la projection de $\hat{Q} n_{1} n_{2} \ldots n_{k} G n_{1} n_{2} \ldots n_{k} n_{k+1}$ sur l'axe $O X$, designons par

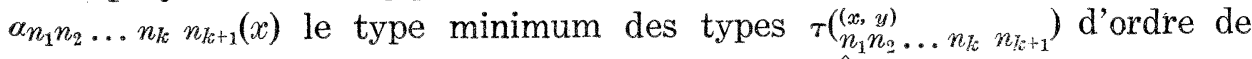
$C_{n_{1} n_{2} \ldots n_{k} n_{k+1}}^{(x, y)}$ lorsque $y$ parcourt l'ensemble $\hat{Q}_{n_{1} n_{2} \ldots n_{k}}^{(x)} G_{n_{1} n_{2} \ldots n_{k} n_{k+1}}$ et par $\hat{Q}_{n_{1} n_{2} \ldots n_{k} n_{k+1}}^{(x)}$ l'ensemble de tous les points $(x, y)$ de $\hat{Q}_{n_{1}} n_{2} \ldots n_{k} \times$ $G n_{1} n_{2} \ldots n_{k} n_{k+1}$ tels qu'on ait $\tau\left(C_{n_{1} n_{2} \ldots n_{k} n_{k+1}}^{(x, y)}\right)=\alpha_{n_{1} n_{2} \ldots n_{k+1}}(x)$. Et enfin, désignons par $\hat{Q}_{n_{1} n_{2} \ldots n_{k} n_{k+1}}$ la somme de tous les ensembles $\hat{Q}_{n_{1} n_{2} \ldots n_{k} n_{k+1}}^{(x)}$ lorsque $x$ parcourt la projection de $\widehat{Q} n_{1} n_{2} \ldots n_{k} G n_{1} n_{2} \ldots n_{k} n_{k+1}$ sur l' axe $O X$.

Nous avons alors d'après les définitions de $\hat{Q} n_{1} n_{2} \ldots n_{k}$ et $\alpha_{n_{1} n_{2} \ldots n_{k}}(x)$ 


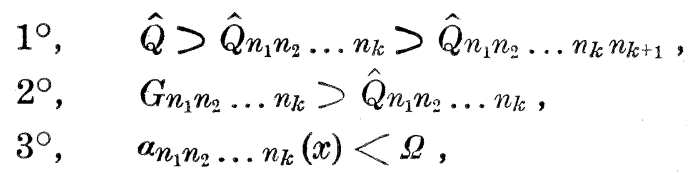

$4^{\circ}$, pour que $(x, y)$ soit un point de $\widehat{Q} n_{1} n_{2} \ldots n_{k}$ (ou $\left.\hat{Q}\right)$, il faut et il suffit que les conditions suivantes soient remplies:
$(\alpha)$,
$\tau\left(C_{n_{1} n_{2} \ldots n_{k}}^{(x, y)}\right)=\alpha_{n_{1} n_{2} \ldots n_{k}}(x)$
$(x)$
(ou $\left.\tau\left(C^{(x, y)}\right)=\alpha(x)\right)$,
$(\beta)$
$(x, y) \in \hat{Q}_{n_{1} n_{2} \ldots n_{k-1}} G_{n_{1} n_{2} \ldots n_{k-1} n_{k}}$
(ou $(x, y) \in R(x, y))$.

Lemme 5. Pour toute suite finie $\left(n_{1}, n_{2}, \ldots, n_{k}\right)$ de nombres naturels, nous avons

$$
\hat{Q}_{n_{1} n_{2} \ldots n_{k}}=Q n_{1} n_{2} \ldots n_{k} \quad \text { et } \quad \hat{Q}=Q .
$$

Démonstration. Nous allons d'abord voir qu'on a $\hat{Q}_{n_{1} n_{2} \ldots n_{k}}>Q n_{1} n_{2} \ldots n k$ et $\hat{Q}>Q$. Prenons un point $(\bar{x}, \bar{y})$ de l'ensemble $Q$. Nous avons alors d'après la définition de $Q(\bar{x}, \bar{y}) \bar{\epsilon} \operatorname{Proj}_{X O Y} M$, d'où nous avons pour tout point $t$ de $R(t)$

$$
(\bar{x}, \bar{y}, t) \bar{\epsilon} \stackrel{(p)}{E}\left(\mathcal{L}_{0}(p) \geqq \stackrel{(2)}{\nu_{0}}(p)+1\right) \quad \text { ou } \quad \stackrel{(1)}{\nu_{0}}(\bar{x}, \bar{y}, t)<\stackrel{(2)}{\nu_{0}}(\bar{x}, \bar{y}, t)+1,
$$

ce qui donne pour tout point $t$ de $R(t) \tau\left(C^{(\bar{x}, \bar{y})}\right)<\tau\left(C^{(\bar{x}, t)}\right)+1$. Or, comme $(\bar{x}, \bar{y})$ est un point du complémentaire analytique $E$, nous avons $\tau\left(C^{(\bar{x}, \bar{y})}\right)<\Omega$ et pour tout point $t$ de $R(t) \tau\left(C^{(\bar{x}, \bar{y})}\right) \leqq \tau\left(C^{(\bar{x}, t)}\right)$, ce qui donne $\tau\left(C^{(\bar{x}, \bar{y})}\right)=\alpha(\bar{x})$, d'où $(\bar{x}, \bar{y})$ est un point de $\hat{Q}$, ou nous avons $\hat{Q}>Q$.

Puis, soit $(\bar{x}, \bar{y})$ un point de l'ensemble $Q n_{1} n_{2} \ldots n_{k}$. Maintenant, supposons par impossible que $(\bar{x}, \bar{y})$ n'appartient pas à l'ensemble $\hat{Q} n_{1} n_{2} \ldots n_{k}$. Puisque $Q n_{1} n_{2} \ldots n_{k}$ est contenu dans $Q,(\bar{x}, \bar{y})$ est un point de $Q$ et par de l'ensemble $\hat{Q}$. Donc, en vertu de $1^{\circ}$ de cette section, nous avons

$$
(\bar{x}, \bar{y}) \in \hat{Q} \quad \text { et } \quad(\bar{x}, \bar{y}) \bar{\in} \hat{Q}_{n_{1}},
$$

ou bien pour un nombre naturel $j(j<k)$

$$
\left(1_{2}\right) \quad(\bar{x}, \bar{y}) \in \hat{Q}_{n_{1} n_{2} \ldots n_{i}}(i=1,2, \ldots, j) \text { et }(\bar{x}, \bar{y}) \bar{\epsilon} \hat{Q}_{n_{1} n_{2} \ldots n_{j} n_{j+1}} .
$$

Or, puisque les deux cas $\left(1_{1}\right)$ et $\left(1_{2}\right)$ sont traités analogiquement, nous ne considérons que le cas $\left(1_{2}\right)$ dans la suite.

Comme $(\bar{x}, \bar{y})$ est un point de $\hat{Q}_{n_{1} n_{2} \ldots n_{i}}(i=1,2, \ldots j)$, nous avons d'après $4^{\circ}$ de cette section

$$
\tau\left(C_{n_{1} n_{2} \ldots n_{i}}^{(\bar{x}, \bar{y})}\right)=\alpha_{n_{1} n_{2} \ldots n_{i}}(\bar{x}) \quad(i=1,2, \ldots j) .
$$

D'ailleurs, Comme $Q n_{1} n_{2} \ldots n_{k}$ est contenu dans le rectangle $G n_{1} n_{2} \ldots n_{k}$ de BAIRE, $(\bar{x}, \bar{y})$ est d'après l'hypothèse un point de $G n_{1} n_{2} \ldots n_{j} n_{j+1}$. Donc, en 
vertu de $\left(1_{2}\right)$ nous avons

$$
(\bar{x}, \bar{y}) \in \hat{Q}_{n_{1} n_{2}} \ldots n_{j} G n_{1} n_{2} \ldots n_{j} n_{j+1},
$$

d'où nous avons $\hat{Q}_{n_{1} n_{2} \ldots n_{j}}^{(x)} G_{n_{1} n_{2} \ldots n_{j} n_{j+1}} \neq 0$, et par suite le domaine de la définition de $\alpha_{n_{1} n_{2} \ldots n_{j} n_{j+1}}(x)$ contient le point $\bar{x}$. Comme nous avons $(\bar{x}, \bar{y}) \bar{\epsilon} \hat{Q} n_{1} n_{2} \ldots n_{j} n_{j+1}$, nous avons selon la proposition $4^{\circ}$ de cette section et $\left(1_{2}\right)$

$$
\tau\left(C_{n_{1} n_{2} \ldots n_{j} n_{j+1}}^{(\bar{x}, \bar{y})}>\alpha_{n_{1} n_{2} \ldots n_{j} n_{j+1}}(\bar{x}) .\right.
$$

Par suite, pour un point $(\bar{x}, \overline{\bar{y}})$ de l'ensemble $\hat{Q}_{n_{1} n_{2} \ldots n_{j}}^{(\bar{x})} G_{n_{1} n_{2} \ldots n_{j} n_{j+1}}$, nous avons

$$
\alpha_{n_{1} n_{2} \ldots n_{j} n_{j+1}}(\bar{x})=\tau\left(C_{n_{1} n_{2} \ldots n_{j} n_{j+1}}^{(\bar{x}, \overline{\bar{y}})}\right)<\tau\left(C_{n_{1} n_{2} \ldots n_{j} n_{j+1}}^{(\bar{x}, \bar{y})}\right) .
$$

Il en résulte que pour un point $(\bar{x}, \bar{y}, \overline{\bar{y}})$ de $R(x, y, t)$ nous avons l'inégalité

$$
\stackrel{(2)}{\nu}_{\nu^{+1}}(\bar{x}, \bar{y}, \bar{y})<\stackrel{(1)}{\nu}_{\nu^{+1}}(\bar{x}, \bar{y}, \bar{y}) \text {. }
$$

En autre part, comme nous avons d'après $1^{\circ}$ de cette $\operatorname{section}(\bar{x}, \bar{y}) \epsilon$ $\hat{Q} n_{1} n_{2} \ldots n_{j} n_{j+1}<\hat{Q} n_{1} n_{2} \ldots n_{i}<\hat{Q}$, nous avons pour $i=1,2, \ldots j+1$

$$
\tau\left(C_{n_{1} n_{2} \ldots n_{i}}^{(\bar{y})}=\alpha_{n_{1} n_{2} \ldots n_{i}}(\bar{x}) \quad \text { et } \quad \tau\left(C^{(\bar{x}, \bar{y})}\right)=\alpha(\bar{x}),\right.
$$

d'où nous avons d'après $\left(\alpha^{*}\right)$

$$
\left.\stackrel{(1)}{\nu_{i}(\bar{x}, \bar{y}}, \overline{\bar{y}}\right)=\stackrel{(2)}{\nu_{i}}(\bar{x}, \bar{y}, \overline{\bar{y}}) \quad(i=0,1,2, \ldots j)
$$

Or, la relation $(\bar{x}, \overline{\bar{y}}) \in \hat{Q} n_{1} n_{2} \ldots n_{j} n_{j+1}$ entraine $(\bar{x}, \overline{\bar{y}}) \in G n_{1} n_{2} \ldots n_{j} n_{j+1}$, et nous avons done d'après (2) $(\bar{x}, \bar{y}, \overline{\bar{y}}) \in D_{j+1}$. Par conséquent, nous avons en vertu de (3) et (4) $(\bar{x}, \bar{y}, \bar{y}) \in M n_{1} n_{2} \ldots n_{j} n_{j+1}$, d'oú nous avons $(\bar{x}, \bar{y}) \bar{\epsilon} Q n_{1} n_{2} \ldots n_{j} n_{j+1}$. Or, puisque nous avons $Q n_{1} n_{2} \ldots n_{k}<Q n_{1} n_{2} \ldots n_{j} n_{j+1},(\bar{x}, \bar{y})$ n'appartient pas à l'ensemble $Q n_{1} n_{2} \ldots n_{k}$, ce qui donne une contradiction. Donc, nous avons $(\bar{x}, \bar{y}) \in \hat{Q}_{n_{1} n_{2} \ldots n_{k}}$ et par suite $Q n_{1} n_{2} \ldots n_{k}<\hat{Q} n_{1} n_{2} \ldots n_{k}$.

Puis, nous allons démontrer qu'on a $\hat{Q}_{n_{1} n_{2} \ldots n_{k}<Q n_{1} n_{2} \ldots n_{k}}$ et $\hat{Q}<Q$. Pour cela, supposons d'abord qu'on ait $(\bar{x}, \bar{y}) \bar{\epsilon} Q$. D'après la définition de $Q$, il existe un point $\bar{t}$ dans $R(t)$ tel qu'on ait $(\bar{x}, \bar{y}, \bar{t}) \in M$, ce qui donne d'après la définition de $M$

$$
\stackrel{(1)}{\nu_{0}}(\bar{x}, \bar{y}, \bar{t}) \geqq \stackrel{(2)}{\nu_{0}}(\bar{x}, \bar{y}, \bar{t})+1 \text {. }
$$

Si l'on a ${ }_{\nu}^{(2)}(\bar{x}, \bar{y}, \bar{t})=\Omega$, nous avons aussi $\stackrel{(1)}{\nu}_{0}(\bar{x}, \bar{y}, \bar{t})=\Omega$, d'où nous avons $\tau\left(C^{(\bar{x}, \bar{y})}\right)=\Omega$. Donc, le point $(\bar{x}, \bar{y})$ n'appartient pas à l'ensemble $\hat{Q}$. Si a ${ }^{(1)}(\bar{x}, \bar{x}, \bar{y}, \bar{t})<\Omega$, nous avons d'après $(5) \stackrel{(1)}{\nu_{0}}(\bar{x}, \bar{y}, \bar{t})>\stackrel{(2)}{\nu_{0}}(\bar{x}, \bar{y}, \bar{t})$, d'où on a 
$\tau\left(C^{(\bar{x}, \bar{y})}\right)>\tau\left(C^{(\bar{x}, \bar{t})}\right)$. Par suite, nous avons $\tau\left(C^{(\bar{x}, \bar{y})}\right)>\alpha(\bar{x})$, et donc $(\bar{x}, \bar{y}) \bar{\epsilon}$ $\hat{Q}$, ce qui donne $\hat{Q} \subset Q$.

Maintenant supposons qu'on ait $(\bar{x}, \bar{y}) \bar{\in} Q n_{1} n_{2} \ldots n_{k}$. Si le point $(\bar{x}, \bar{y})$ n'appartient pas à $Q$, nous avons d'après le résultat obtenu plus haut $(\bar{x}, \bar{y}) \bar{Q} \hat{Q}$. Or, comme $\hat{Q} n_{1} n_{2} \ldots n_{k}$ est un sous-ensemble de $\hat{Q}$, nous avons $(\bar{x}, \bar{y}) \bar{\epsilon} \hat{Q} n_{1} n_{2} \ldots n_{k}$. Puis, considérons le cas où $(\bar{x}, \bar{y})$ appartient á $Q$. Nous avons alors

$$
(\bar{x}, \bar{y}) \in Q \quad \text { et } \quad(\bar{x}, \bar{y}) \bar{\epsilon} Q n_{1},
$$

ou bien pour un nombre naturel $j(j<k)$

$$
(\bar{x}, \bar{y}) \in Q n_{1} n_{2} \ldots n_{i}(i=1,2, \ldots j) \quad \text { et } \quad(\bar{x}, \bar{y}) \bar{\epsilon} Q n_{1} n_{2} \ldots n_{j} n_{j+1} .
$$

Comme nous pouvons traiter analogiquement les deux cas $\left(6_{1}\right)$ et $\left(6_{2}\right)$, nous ne considérons que le cas $\left(6_{2}\right)$ dans la suite. Or, puisque nous avons d'après le résultat obtenu plus haut $Q n_{1} n_{2} \ldots n_{i}<\hat{Q} n_{1} n_{2} \ldots n_{i}(i=1,2, \ldots j)$, nous avons $(\bar{x}, \bar{y}) \in \hat{Q} n_{1} n_{2} \ldots n_{i}(i=1,2, \ldots j)$ et $(\bar{x}, \bar{y}) \in \hat{Q}$, ce qui entraine pour $i=1,2, \ldots j$

$$
\left(\alpha^{* *}\right) \quad \tau\left(C_{n_{1} n_{2} \ldots n_{i}}^{(\bar{y}, \bar{y})}=\alpha_{n_{1} n_{2} \ldots n_{i}}(\bar{x}) \quad \text { et } \quad \tau\left(C^{(\bar{x}, \bar{y})}\right)=\alpha(\bar{x}) .\right.
$$

En autre part, comme on a $(\bar{x}, \bar{y}) \bar{\epsilon} Q n_{1} n_{2} \ldots n_{j} n_{j+1}=G n_{1} n_{2} \ldots n_{j} n_{j+1}-P_{X} j_{X O Y}$ $M n_{1} n_{2} \ldots n_{j} n_{j+1}$, on peut distinguer les deux cas.

Le premier cas. Si l'on a $(\bar{x}, \bar{y}) \bar{\epsilon} G n_{1} n_{2} \ldots n_{j} n_{j+1}$, nous avons aussi $(\bar{x}, \bar{y}) \bar{\epsilon} G n_{1} n_{2} \ldots n_{k}$ et par suite $(\ddot{x}, \bar{y}) \bar{\epsilon} \hat{Q} n_{1} n_{2} \ldots n_{k}$.

Le deuxième cas. Si le point $(\bar{x}, \bar{y})$ est un point de $G n_{1} n_{2} \ldots n_{j} n_{j+1}$, nous avons d'après l'hypothèse $(\bar{x}, \bar{y}) \in \operatorname{Proj}_{X O Y} M_{n_{1} n_{2} \ldots n_{j} n_{j+1}}$ et par suite, il existe un point $(\bar{x}, \bar{y}, \bar{t})$ de $R(x, y, t)$ tel qu'on ait $(\bar{x}, \bar{y}, \bar{t}) \in M n_{1} n_{2} \ldots n_{j} n_{j+1}$. Or, comme nous avons $(\bar{x}, \bar{y}) \in Q n_{1} n_{2} \ldots n_{i}$, nous avons d'après la définition du $Q n_{1} n_{2} \ldots n_{i}(\bar{x}, \bar{y}, \bar{t}) \bar{\epsilon} M_{n_{1} n_{2} \ldots n_{i}}$, d'où on a en vertu de la définition de $M n_{1} n_{2} \ldots n_{j} n_{j+1}$

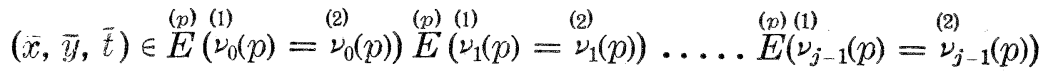

$$
\begin{aligned}
& \left.\times \stackrel{(p)}{E}{\stackrel{(1)}{\nu_{j}}(p)}^{\left(\stackrel{(2)}{\nu_{j}}(p)\right)} \stackrel{(p)}{E}{\stackrel{(1)}{\nu_{j+1}}}(p) \geqq \stackrel{(2)}{\nu_{j+1}}(p)+1\right) D_{j+1},
\end{aligned}
$$

ce qui donne les résultats suivants

$5^{\circ},(\bar{x}, \bar{y}, \bar{t}) \in D_{j+1}$ et par suite, nous avons $(\bar{x}, \bar{t}) \in G n_{1} n_{2} \ldots n_{j} n_{j+1}$, ${ }_{(2)} 6^{\circ},(\bar{x}, \bar{y}, \bar{t}) \in \underline{E}\left(\nu_{i}(p)=\nu_{i}(p)\right)(i=1,2, \ldots j)$, ce qui entraine $\nu_{i}(\bar{x}, \bar{y}, \bar{t})$ $=\nu_{i}(\bar{x}, \bar{y}, \bar{t})$,

$$
7^{\circ},(\bar{x}, \bar{y}, \bar{t}) \in \stackrel{(p)}{E^{(}}\left(\nu_{j+1}^{()}(p) \geq \stackrel{(2)}{\nu_{j+1}}(p)+1\right) .
$$


Or, d'après l'hypothèse sur le point $(\bar{x}, \bar{y})$, nous avons $(\bar{x}, \bar{y}) \in Q n_{1} n_{2} \ldots n_{i}$ et par suite, nous avons selon $\left(\alpha^{* *}\right)$ et $6^{\circ}$

$$
\tau\left(C_{n_{1} n_{2} \ldots n_{i}}^{(\bar{x})}\right)=\alpha_{n_{1} n_{2} \ldots n_{i}}(\bar{x}) \quad(i=1,2 \ldots j) \quad \text { et } \quad \tau\left(C^{(\bar{x}, \bar{t})}\right)=\alpha(\bar{x}) .
$$

Nous avons d'après $(7)(\bar{x}, \bar{t}) \in \hat{Q}$. Or, nous avons $(\bar{x}, \bar{t}) \in G n_{1} n_{2} \ldots n_{j} n_{j+1} \subset G n_{1}$ et par consequent $(\bar{x}, \bar{t}) \in \hat{Q} G n_{1}$, ce qui donne de (7) et la proposition $4^{\circ}$ de cette section $(\bar{x}, \bar{t}) \in \hat{Q} n_{1}$. Or, comme $(\bar{x}, \bar{t})$ est un point de $G n_{1} n_{2} \ldots n_{j} n_{j+1}$ et par suite de $G n_{1} n_{2}$, nous avons $(\bar{x}, \bar{t}) \in \widehat{Q} n_{1} G n_{1} n_{2}$, d'où nous avons d'après (7) et la proposition $4^{\circ}$ de cette section $(\bar{x}, \bar{t}) \in \hat{Q}_{n_{1} n_{2}}$. De même, nous pouvons démontrer de proche en proche qu'on a $(\bar{x}, \bar{t}) \in \hat{Q} n_{1} n_{2} \ldots n_{i}(i=1$ $1, \ldots j$ ), ce qui entraine

$$
(\bar{x}, \bar{t}) \in \hat{Q}_{n_{1} n_{2} \ldots n_{j}} G_{n_{1} n_{2} \ldots n_{j} n_{j \div 1}} \cdot
$$

Or, nous avons d'après $\left(7^{\circ}\right)$ de cette section $\left.\stackrel{(1)}{\nu}_{j+1}^{(\bar{x}}, \bar{y}, \bar{t}\right) \geqq \stackrel{(2)}{\nu}_{j+1}(\bar{x}, \bar{y}, \bar{t})+1$, c'est-à-dire, nous avons

$$
\tau\left(C_{n_{1} n_{2} \ldots n_{j} n_{j+1}}^{(\bar{x}, \bar{y})}\right) \geq \tau\left(C_{n_{1} n_{2} \ldots n_{j} n_{j+1}}^{(\bar{x}, \bar{t})}\right)+1 .
$$

Et, nous avons d'après (7) $\nu\left(C_{n_{1} n_{2}}^{(\bar{x}, \bar{t})} \ldots n_{j} n_{j+1}\right) \leqq \tau\left(C^{(\bar{x}, \bar{t})}\right)=\alpha(\bar{x})<\Omega . \quad$ Par conséquent nous avons d'après (8) et (9) $\tau\left(C_{n_{1} n_{2} \ldots n_{j} n_{j+1}}^{(\bar{x}, \bar{y})}>\alpha n_{1} n_{2} \ldots n_{j} n_{j+1}(\bar{x})\right.$, ce qui donne $(\bar{x}, \bar{y}) \bar{\epsilon} \hat{Q} n_{1} n_{2} \ldots n_{j} n_{j+1}$, d'où nous avons aussi $(\bar{x}, \bar{y}) \bar{\epsilon} \hat{Q} n_{1} n_{2} \ldots n_{k}$.

Par consequent nous avons dans tous les cas $(\bar{x}, \bar{y}) \bar{\epsilon} \hat{Q} n_{1} n_{2} \ldots n_{k}$, et donc $\hat{Q} n_{1} n_{2} \ldots n_{k}<Q n_{1} n_{2} \ldots n_{k}$, d'où nous avons aussi $Q=\hat{Q}$ et $Q n_{1} n_{2} \ldots n_{k}$ $=\hat{Q} n_{1} n_{2} \ldots n_{k}$.

C. Q. F. D.

10. Une propriété des ensembles $Q n_{1} n_{2} \ldots n_{k}$. Étant donné un crible $C=\sum_{n_{1} n_{2} \ldots n_{k}} G n_{1} n_{2} \ldots n_{k} \times\left(r n_{1} n_{2} \ldots n_{k}\right)$ complètement régulier (de classe $n$ ), où les rectangles $G n_{1} n_{2} \ldots n_{k}$ de BAIRE et les nombres rationnels. $r n_{1} n_{2} \ldots n_{k}$ satisfont aux conditions données dans le lemme 3, nous définirons les ensembles $Q$ et $Q n_{1} n_{2} \ldots n_{k}\left(k, n_{k}=1,2, \ldots\right)$, comme nous avons plus haut. On peut alors démontrer un lemme suivant utilisé dans la suite.

Lemme 6. Étant donné un point $\bar{x}$ de $\underset{\infty}{R}(x)$, si l'on a $Q_{n_{1} n_{2} \ldots n_{k}}^{(\bar{x})} \neq 0$ $(k=1,2, \ldots)$, nous avons $\prod_{k=1}^{\infty} Q n_{1} n_{2} \ldots n_{k}=\prod_{k=1}^{\infty} G n_{1} n_{2} \ldots n_{k} \neq 0$.

Démonstration. Comme nous avons $Q n_{1} n_{2} \ldots n_{k}<G n_{1} n_{2} \ldots n_{k}$, nous avons d'après l'hypothèse $G_{n_{1} n_{2} \ldots n_{k}}^{(\bar{x})} \neq 0(k=1,2, \ldots)$, d'où la partie commune $\prod_{k=1}^{\infty} G_{n_{1} n_{2}}^{(\bar{x})} \ldots n_{k}$ est non vide et par suite précisément un point, on peut représenter ce point sous la forme $(\bar{x}, \bar{y})$. Nous pouvons alors voit que $(\bar{x}, \bar{y})=\prod_{k=1}^{\infty} Q n_{1} n_{2} \ldots u_{k}$. Pour cela, démontrons les propositions suivantes. 
$1^{\circ}$. Nous avons $\alpha_{n_{1} n_{2} \ldots n_{i}}(\bar{x})<\alpha_{n_{1} n_{2} \ldots n_{j}}(\bar{x})$ ou bien $>\alpha_{n_{1} n_{2} \ldots n_{j}}(\bar{x})$, suivant qu'on ait $r n_{1} n_{2} \ldots n_{i}<r n_{1} n_{2} \ldots n_{j}$ ou bien $<r n_{1} n_{2} \ldots n_{j}$.

En effet, prenons un nombre naturel $k$ tel qu'on ait $k>i, j$. Comme nous avons $Q_{n_{1} n_{2} \ldots n_{k}}^{(\bar{x})} \neq 0$, on peut choisir parmi $Q_{n_{1} n_{2} \ldots n_{k}}^{(\bar{x})}$ un point, désignons par $(\bar{x}, \bar{y})$ ce point. Puisque nous avons $Q n_{1} n_{2} \ldots n_{k}<Q n_{1} n_{2} \ldots n_{i}$ et $\angle Q n_{1} n_{2} \ldots n_{j}$, le point $(\bar{x}, \bar{y})$ est contenu dans les ensembles $Q n_{1} n_{2} \ldots n_{j}$ et $Q n_{1} n_{2} \ldots n_{j}$ en même temps, et nous avons donc

$$
\begin{gathered}
(\bar{x}, \bar{y}) \in G_{n_{1} n_{2} \ldots n_{i}} \quad \text { et } \quad \in G_{n_{1} n_{2} \ldots n_{j}}, \\
\tau\left(C_{n_{1} n_{2} \ldots n_{i}}^{(\bar{x}, \bar{y})}=\alpha_{n_{1} n_{2} \ldots n_{i}}(\bar{x}) \quad \text { et } \quad \tau\left(C_{n_{1} n_{2} \ldots n_{j}}^{(\bar{x}, \bar{y})}\right)=\alpha_{n_{1} n_{2} \ldots n_{j}}(\bar{x}),\right.
\end{gathered}
$$

d'où nous avons $\alpha_{n_{1} n_{2} \ldots n_{i}}(\bar{x})<\alpha_{n_{1} n_{2} \ldots n_{j}}(\bar{x})$ ou $>\alpha_{n_{1} n_{2} \ldots n_{j}}(\bar{x})$ suivant qu'on ait $r n_{1} n_{2} \ldots n_{i}<r u_{1} n_{2} \ldots n_{j}$ ou $>r n_{1} n_{2} \ldots n_{j}$.

$2^{\circ}$. Nous avons $\alpha_{n_{1} n_{2} \ldots n_{k}}(\bar{x})<\alpha(\bar{x})$.

En effet, prenons un point $(\bar{x}, y)$ dans l'ensemble $Q n_{1} n_{2} \ldots n_{k}$. Comme nous avons $Q n_{1} n_{2} \ldots n_{k}<Q$, nous avons

$$
\alpha_{n_{1} n_{2} \ldots n_{k}}(\bar{x})=\tau\left(C_{n_{1} n_{2} \ldots n_{k}}^{(\bar{x}, y)}\right)<\tau\left(C^{(\bar{x}, y)}\right)=\alpha(\bar{x}),
$$

d'où nous avons $\alpha_{n_{1} n_{2} \ldots n_{k}}(\bar{x})<\alpha(\bar{x})$.

$3^{\circ}$. L'ensemble $\left\{\alpha_{n_{1}} n_{2} \ldots n_{k}(\bar{x})\right\}(k=1,2, \ldots)$ de nombres ordinaux est bien ordonné de type $\alpha(\bar{x})$ suivant la grandeur des nombres ordinaux.

En effet, selon la définition $C=\sum_{n_{1} n_{2} \ldots n_{k}} G n_{1} n_{2} \ldots n_{k} \times\left(r n_{1} n_{2} \ldots n_{k}\right)$ est complètement régulier, $C^{(\bar{x}, \bar{y})}$ est l'ensemble de nombres $r n_{1} n_{2} \ldots n_{k}$ $(k=1,2, \ldots)$ rationnels, et d'après la définition $\mathrm{du} \bar{x}$, le type d'ordre de l'ensemble $\left\{r_{n_{1}} n_{2} \ldots n_{k}\right\}(k=1,2, \ldots)$ n'est pas inférieur à $\alpha(\bar{x})$. Il en resulte que l'ensemble $\left\{\alpha_{n_{1}} n_{2} \ldots n_{k}(x)\right\}(k=1,2, \ldots)$ de nombres ordinaux est d'après la proposition $1^{\circ}$ de cette section bien ordonné de type $\geqq \alpha(\bar{x})$ suivant la grandeur des nombres ordinax. Or, d'après la proposition $2^{\circ}$ de cette section, l'ensemble $\left\{\alpha_{n_{1} n_{2} \ldots n_{k}}(\bar{x})\right\}(k=1,2, \ldots)$ de nombres ordinaux ne contient que les nombres ordinaux inferieurs à $\alpha(\bar{x})$, d'où l'ensemble $\left\{\alpha_{\left.n_{1} n_{2} \ldots n_{k}(\bar{x})\right\}}(k=1,2, \ldots)\right.$ est bien ordonné de type $\leqq \alpha(\bar{x})$ suivant la grandeur de nombres ordinaux. L'ensemble $\left\{\alpha_{\left.n_{1} n_{2} \ldots n_{k}(\bar{x})\right\}}\right.$ est donc bien ordonné de type $\alpha(\bar{x})$.

$$
4^{\circ} . \quad(\bar{x}, \bar{y}) \in Q^{(\bar{x})} \quad \text { et } \quad(\bar{x}, \bar{y}) \in Q_{n_{1} n_{2} \ldots n_{k}}^{(\bar{x})} \quad(k=1,2, \ldots) .
$$

En effet, d'après la proposition précédent l'ensemble $\left\{\alpha_{n_{1} n_{2} \ldots n_{l}(\bar{x})}\right\}$ $(k=1,2, \ldots)$ est bien ordonné du type $\alpha(\bar{x})$. Or, l'ensemble a de tous les nombres ordinaux $\alpha$ tel qu'on ait $\alpha<\alpha(\bar{x})$ est aussi bien ordonné du 
type $\alpha(\bar{x})$ et par suite on peut définir une application semblable $T$ qui

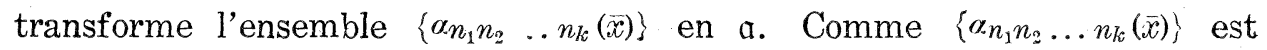
un sous-ensemble de $\mathfrak{a}$, en désignant par $\alpha_{k}^{*}$ un nombre ordinal de a qui correspond à $\alpha_{n_{1} n_{2} \ldots n_{k}}(\bar{x})$ par l'application semblable $T$, nous avons

$$
\alpha_{n_{1} n_{2} \ldots n_{k}}(\bar{x}) \geqq \alpha_{k}^{*} \quad(k=1,2, \ldots) .
$$

Or, en autre part, on peut démontrer par l'induction transfinie sur $\alpha_{k}^{*}$

$$
\tau\left(C_{n_{1} n_{2} \ldots n_{k}}^{(\bar{x}, \bar{y})}\right)=\alpha_{k}^{*} \quad(k=1,2, \ldots) .
$$

Prenons d'abord $\alpha_{k}^{*}$ tel qu'on ait $\alpha_{k}^{*}=0 . \quad \alpha_{n_{1} n_{2}} \ldots n_{k}(\bar{x})$ est alors un nombre ordinal plus petit dans $\left\{\alpha_{n_{1}} n_{2} \ldots n_{k}(\tilde{x})\right\}$, c'est-à-dire, $\alpha_{n_{1} n_{2} \ldots n_{k}}$ $(\bar{x})<\alpha_{n_{1} n_{2}} \ldots n_{j}(\bar{x})$ pour $j \neq k$, d'où nous avons d'après la proposition $1^{\circ}$ de cette section $r_{n_{1}} n_{2} \ldots n_{k}<r n_{1} n_{2} \ldots n_{j}$ pour $j \neq k$, Il en resulte qu'on a $\tau\left(C_{n_{1} n_{2} \ldots n_{k}}^{(\bar{x}, \bar{y})}\right)=0=\alpha_{k}^{*}$. Étant donné un nombre ordinal $\alpha$ tel qu'on ait $\alpha<\alpha(\bar{x})$, supposons que $\alpha_{k}^{*}<\alpha$ entraine $\tau\left(C_{n_{1} n_{2} \ldots n_{k}}^{(\bar{x}, \bar{y})}\right)=\alpha_{k}^{*}$, et considérons un nombre ordinal $\alpha_{k_{0}}^{*}$ tel qu'on ait $\alpha_{k_{0}}^{*}=\alpha$. D'après la définition, l'ensemble $a^{*}$ de tous les nombres ordinaux $\alpha_{j}^{*}$ tels qu'on ait $\alpha_{j}^{*}<\alpha_{k_{0}}^{*}=\alpha$ est bien ordonné du type $\alpha$ suivant la grandeur des nombres ordinaux. Or; comme la relation $\alpha_{i}^{*}<\alpha_{j}^{*}$, où $\alpha_{i}^{*}$ et $\alpha_{j}^{*}$ sont des nombres ordinaux

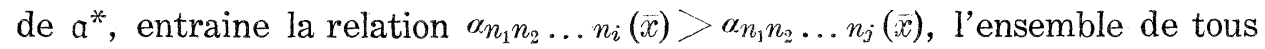
les nombres ordinaux $\alpha_{n_{1} n_{2} \ldots n_{j}}(\bar{x})$ tels qu'on ait $\alpha_{j}^{*}<\alpha_{k_{0}}^{*}=\alpha$ est bien ordonné du type $\alpha$. Or, puisque la relation $r n_{1} n_{2} \ldots n_{i}>r n_{1} n_{2} \ldots n_{j}$ est

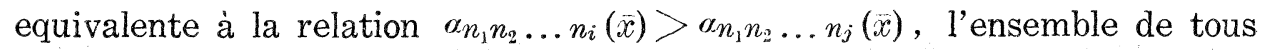
les nombres rationnels $r n_{1} n_{2} \ldots n_{j}$ tels qu'on ait $\alpha_{j}^{*}<\alpha_{k_{0}}^{*}=\alpha$, c'est-à-dire, $r n_{1} n_{2} \ldots n_{j}<r n_{1} n_{2} \ldots n_{k 0}$ est bien ordonné du type $\alpha$, d'où nous avons $\tau\left(C_{n_{1} n_{2} \ldots n_{k_{0}}}^{(\bar{x}, \bar{y})}\right)=\alpha=\alpha_{k_{0}}^{*}$. Nous avons donc par l'induction transfinie $\tau\left(C_{n_{1} n_{2} \ldots n_{k}}^{(\bar{x}, \bar{y})}\right)=\alpha_{k}^{*}$. Maintenant, démontrons qu'on a $(\bar{x}, \bar{y}) \in Q$ et $(\bar{x}, \bar{y}) \in Q n_{1} n_{2} \ldots n_{k}(k=1,2, \ldots)$. Selon la définition du crible $C$ complètement régulier, $C(\bar{x}, \bar{y})$ est l'ensemble de nombres rationnels $r n_{1} n_{2} \ldots n_{k}$ $(k=1,2, \ldots)$. Or, comme les deux ensembles $\left\{\alpha_{n_{1} n_{2} \ldots n_{k}}(\bar{x})\right\}(k=1,2, \ldots)$ et $\left\{r n_{1} n_{2} \ldots n_{k}\right\}(k=1,2, \ldots)$ sont d'après la proposition $1^{\circ}$ de cette section semblables deux-à-deux et comme l'ensemble $\left\{\alpha_{n_{1}} n_{2} \ldots n_{k}(\bar{x})\right\}(k=1,2, \ldots)$ est d'après la proposition $3^{\circ}$ de cette section bien ordonné de type $\alpha(\bar{x})$, l'ensemble $\left\{r n_{1} n_{2} \ldots n_{k}\right\}(k=1,2, \ldots)$ est aussi bien ordonné de type $\alpha(\bar{x})$, c'est-à-dire, $\tau(C(\vec{x}, \bar{y}))=o(\bar{x})$, et par suite, nous avons $(\bar{x}, \bar{y}) \in Q$. Puisque nous avons $(\bar{x}, \bar{y}) \in G n_{1}$, nous avons $(\bar{x}, \bar{y}) \in Q G n_{1}$, ce qui entraine $\tau\left(C_{n_{1}}^{(\bar{x}, \bar{y})}\right) \geqq \alpha_{n_{1}}(\bar{x})$. Nous avons donc d'après (1) et (2) $\tau\left(C_{n_{1}}^{(\bar{x}, \bar{y})}\right) \geqq \alpha_{n_{1}}(\bar{x}) \geqq \alpha_{1}^{*}$ $=\tau\left(C_{n_{1}}^{(\bar{x}, \bar{y})}\right)$, d'où nous avons $\tau\left(C_{n_{1}}^{(\bar{x}, \bar{y})}\right)=\alpha_{n_{1}}(\bar{x})$, et par suite nous avons 
$(\bar{x}, \bar{y}) \in Q n_{1}$. Comme on a $(\bar{x}, \bar{y}) \in Q n_{1} G n_{1} n_{2}$, nous avons $\tau\left(C_{n_{1} n_{2}}^{(\bar{x}, \bar{y})}\right) \geqq \alpha_{n_{1} n_{2}}(\bar{x})$, ce qui donne d'après (1) et (2) $\tau\left(C_{n_{1} n_{2}}^{(\bar{x}, \bar{y})}\right) \geq \alpha_{n_{1} n_{2}}(\bar{x}) \geqq \alpha_{2}^{*}=\tau\left(C_{n_{1} n_{2}}^{(\bar{x}, \bar{y})}\right)$, d'où nous avons $\tau\left(C_{n_{1} n_{2}}^{(\bar{x}, \bar{y})}\right)=\alpha_{n_{1} n_{2}}(\bar{x})$. Par consequent, nous avons $(\bar{x}, \bar{y}) \in Q n_{1} n_{2}$. De même on peut voir de proche en proche qu'on a $(\bar{x}, \bar{y}) \in Q n_{1} n_{2} \ldots n_{k}$.

Il est alors évident d'après la proposition $4^{\circ}$ de cette section qu'on a $(\bar{x}, \bar{y}) \in \prod_{k=1}^{\infty} Q n_{1} n_{2} \ldots n_{k}$.

C. Q. F. D.

11. La définition d'un complémentaire analytique latéralement fermé. Maintenant, posons

$$
S_{k}=\sum_{n_{1} n_{2} \ldots n_{k}} Q n_{1} n_{2} \ldots n_{k} \quad \text { et } \quad S=\prod_{k=1}^{\infty} S_{k}
$$

Comme $Q n_{1} n_{2} \ldots n_{k}$ sont les complémentaires analytiques, $S$ est aussi le complémentaire analytique. Or, puisque $Q n_{1} n_{2} \ldots n_{k}$ sont les sous-ensembles de $Q$ et par suite de $E$, nous avons $S<E$. Nous appellons $S$ un complémentaire analytique latéralement fermé.

Lemme 7. Pour tout point $\bar{x}$ de $R(x)$ tel qu'on ait $E^{(\bar{x})} \neq 0, S^{(\bar{x})}$ est non vide et fermé dans $R(y)$.

Démonstration. Comme nous avons $E^{(\bar{x})} \neq 0$, nous avons d'après la définition de $Q$ aussi $Q^{(\bar{x})} \neq 0$. Par suite, on peut choisir d'après le lemme 3 un rectangle $G_{n_{1}}$ de BAIRE tel qu'on ait $G n_{1} Q^{(\bar{x})} \neq 0$, d'où nous avons selon la définition de $Q n_{1}$ aussi $Q_{n_{1}}^{(\bar{x})} \neq 0$. Il existe donc d'après le lemme 3 un rectangle $G n_{1} n_{2}$ de Baire tel qu'on ait $G n_{1} n_{2} Q_{n_{1}}^{(\bar{x})} \neq 0$, ce qui entraine d'après la définition de $Q n_{1} n_{2}$ aussi $Q_{n_{1} n_{2}}^{(\bar{x})} \neq 0$. De même, on peut définir de proche en proche les nombres naturele $\left\{n_{k}\right\}(k=3,4,5, \ldots)$ tels qu'on ait $Q_{n_{1} n_{2} \ldots n_{k}}^{(\bar{x})} \neq 0$ et par suite $G_{n_{1} n_{2} \ldots n_{k}}^{(\bar{x})} \neq 0$. Maintenant prenons un point $(\bar{x}, \bar{y})$ donné comme le produit $\prod_{k=1}^{\infty} G n_{1} n_{2} \ldots n_{k}$. Nous avons alors d'après le lemme $6(\bar{x}, \bar{y})=\prod_{k=1}^{\infty} Q n_{1} n_{2} \ldots n_{k}<S$, d'où nous avons $S^{(\bar{x})} \neq 0$.

Puis, nous démontrerons que $S^{(\bar{x})}$ est fermé dans $R(y)$. Supposons que le point $(\bar{x}, \bar{y})$ soit un point d'accumulation de l'ensemble $S^{(\bar{x})}$ et qu'on ait $(\bar{x}, \bar{y}) \in R(x, y)$. D'après la définition de $G n_{1} n_{2} \ldots n_{k}$, on peut définir une suite $\left\{n_{k}\right\}(k=1,2, \ldots)$ de nombres naturels telle qu'on ait $(\bar{x}, \bar{y})=\prod_{k=1}^{\infty} G n_{1} n_{2} \ldots n_{k}$. Or, puisque $G n_{1} n_{2} \ldots n_{k}$ est un voisinage du point $(\bar{x}, \bar{y})$ dans $R(\bar{x}, y)$, l'ensemble $G n_{1} n_{2} \ldots n_{k}$ contient un point de $S^{(\bar{x})}$ et par suite de $S_{k}^{(\bar{x})}$, d'oú nous avons $Q_{n_{1} n_{2} \ldots n_{k}}^{(\bar{x})}=S_{k}^{(\bar{x})} G n_{\infty} n_{2} \ldots n_{k} \neq 0$ $(k=1,2, \ldots)$. Nous avons alors d'après le lemme $6(\bar{x}, \bar{y})=\prod_{k=1}^{\infty} Q n_{1} n_{2} \ldots n_{k}$, 
d'où nous avons $(\bar{x}, \bar{y}) \in S_{k}^{(\bar{x})}(k=1,2, \ldots)$ ou $(\bar{x}, \bar{y}) \in S^{(\bar{x})}$. Il en resulte que $S^{(\bar{x})}$ est fermé dans $R(y)$.

C. Q. F. D.

Lemme 8. Si l'ensemble $E$ est donné effectivement l'ensemble $S$ est aussi un complémentaire effectivemnt analytique, et est contenu dans l'ensemble des points d'index minimum par rapport au crible complètement régulier qui définit $E$.

En effet, si l'ensemble $E$ est donné effectivement, on peut définir effectivement un crible $C$ complètement régulier qui définit $E$. D'après la section 6 , les ensembles $Q n_{1} n_{2} \ldots n_{k}\left(k, n_{k}=1,2, \ldots\right)$ sont des complèmentaires effectivement analytiques, et par suite l'ensemble $S$ est aussi de même. En autre part, les ensembles $Q_{n_{1}} n_{2} \ldots n_{k}$ sont contenus dans l'ensemble des points d'index minimum par rapport à $C$, et donc $S$ est contenu dans le même ensemble.

C. Q. F. D.

12. La construction d'ensemble uniformisateur. Maintenant, nous allons définir les complémentaires analytiques latéralement fermés $T_{k}(k=1,2, \ldots)$ comme il suit. Étant donné un complémentaire $E$ effectivement analytique dans $R(x, y)$, nous définirons effectivement un crible $C_{1}$ complètement régulier de classe 1 qui définit $E$. En se servant de ce crible $C_{1}$, nous définirons un complémentaire analytique latéralement fermé et nous désignerons par $T_{1}$ ce ensemble. Or, comme $T_{1}$ est d'après les lemmes 4 et 8 un complémentaire effectivement analytique, on peut définir effectivement un crible $C_{2}$ complètement régulier de classe 2 qui définit $T_{1}$. En utilisant le crible $C_{2}$ ainsi obtenu, nous définirons un complémentaire analytique latéralement fermé et nous désignerons par $T_{2}$ cet ensemble. D'une manière générale, $T_{k}(k=1,2, \ldots n)$ étant effectivement définis, prenons effectivement un crible $C_{n+1}$ complètment régulier de classe $n+1$ qui défnit $T_{n}$. Désignons par $T_{n+1}$ le complémentaire analytique latéralement fermé donné par le crible $C_{n+1}$. Maintenant, posons

$$
U=\prod_{n=1}^{\infty} T_{n}
$$

Selon la définition de $T_{n}$, l'ensembie $U$ est contenu dans $E$. Prenons un point $\bar{x}$ sur l'axe $O X$ tel qu'on ait $E^{(\bar{x})} \neq 0$, l'ensemble $T_{n}^{(\bar{x})}$ est alors fermé non vide dans l'ensemble $R(y)$ et est contenu dans l'ensemble des points d'index minimum par rapport à $C_{n}$, et par suite dans un intervalle de BAIRE d'ordre $n$. Désignons par $I_{n}$ cet intervalle de BAIRE. Or, comme nous avons $T_{n}^{(\bar{x})}>T_{n+1}^{(\bar{x})}(n=1,2, \ldots)$, nous avons $I_{n}>I_{n+1}$ et par suite le produit $\prod_{k=1}^{\infty} I_{n}$ est précisément un point. Désignons par $(\bar{x}, \bar{y})$ ce point ainsi obtenu. Comme l'ensemble $T_{n}^{\overline{(x)}}$ n'est pas vide, $T_{1}^{(\bar{x})}$ contient au moins un point de l'intervalle $I_{n}$. Or, $T_{1}^{(\bar{x})}$ est fermé dans $R(y)$, 
et donc $(\bar{x}, \bar{y})$ appartient à l'ensemble $T_{1}^{(x)}$. De même, on peut démontrer qu'on a $(\bar{x}, \bar{y}) \in T_{n}^{(\bar{x})}(n=2,3, \ldots)$, ce qui entraine $(\bar{x}, \bar{y}) \in \prod_{k=1}^{\infty} T_{n}^{(\bar{x})}=U^{(\bar{x})}$. Or, comme nous avons $T_{n}^{\overline{(x)}}<I_{n}(n=1,2, \ldots)$, $U^{(\overline{x)}}$ est précisément un point. Il en resulte que $U$ est un ensemble uniformisateer de $E$. Or, puisque $T_{n}(n=1,2, \ldots)$ sont des complémentaires effectivement analytiques, l'ensemble uniformsateur $U$ est aussi un complémentaire effectivement analytique.

Remarque. Le théorème de l'uniformisation est celui qui concerne des complémentaires analytiques plans. De même, comme nous avons fait précedemment, on peut établir un théorème sur l'uniformisation des complémentaires analytiques contenus dans un espace à quelques dimensions.

\section{Quelques applications du théorème de l'uniformisation sur des ensembles projectifs de la seconde classe.}

13. La projection uniforme des complémentaires analytiques. Soit $E$ un ensemble projectif de la seconde classe contenu dans un espace $X_{1} O X_{2} X_{3} \ldots X_{n}$ à $n$ dimensions. On peut alors choisir dans l'espace $X_{1} O X_{2} \ldots X_{n} T$ un complémentaire analytique $C$ dont la projection sur le plan $t=0$ est l'ensemble $E$. Or, on peut uniformiser, d'après le théorème de l'uniformisation, $C$ par un complémentaire analytique $U$, et l'ensemble $E$ est donc la projection uniforme du complémentaire analytique $U$. Nous avons done le

Théorème 1. Tout ensemble projectif de la seconde classe peut être donné comme la projection uniforme d'un complémentaire analytique.

Ce théorème contient un théorème de M. S. Mazurkiewiz ( ${ }^{15}$ ) comme le cas spécial, et 'selon ce théorème, on voit sans peine que la classe de tous les ensembles donnés comme la projection uniforme des complémentaires analytiques coincide avec celle des ensembles projectifs de là seconde classe.

M. P. NovikofF a démontré dans sa note $\left({ }^{16}\right)$ quelques théorèmes sur la séparation des ensembles projectifs de la classe $\left(A_{2}^{\prime}\right)$. Grâce à ce théorème, on peut donc démontrer les théorèmes suivants $\left({ }^{17}\right)$ concernent les séparations des ensembles projectifs de la seconde classe.

(15) S. Mazurkiewicz; Sur une proprété des ensembles $C(A)$, Fund. Math., 10 (1917), 172-174.

(16) P. Novikoff, loc. cit., (6), 3-16.

(17) P. Novikoff, Sur la separabilité des ensembles projectifs de seconde classe, Fund. Math., 25 (1935), 459-466. 
Théorème A. Deux ensembles complémentaires aux ensembles de projectifs de la seconde classe sans points communs sont séparables au moyen des ensembles projectifs de la classe $\left(B_{2}\right)$.

Théorème B. Si l'on supprime de deux ensembles projectifs de la seconde classe leur partie commune, les restantes sont séparables, simultanément au moyen des ensembles projectifs de la seconde classe.

14. Une proposition de M.N. LusiN. $E$ étant un ensemble linéaire projectif de la seconde classe. On peut alors choisir dans le plan $X O Y$ un complémentaire analytique $C$ tel que la projection uniforme de $C$ sur l'axe $O X$ soit $E$. Or, d'après un théorème de M. N. Lusin, ( $\left.{ }^{18}\right)$ on peut décomposer $C$ en une classe de puissance $\mathbf{\Sigma}_{1}$ d'ensembles measurables $(B)$ disjoints, et les projections de ces ensembles mesurables $(B)$ ainsi obtenus sur l'axe $O X$ sont aussi mesurables $(B)$. Nous avons donc le

Théorème 2. Tout ensemble projectif de la seconde classe est la somme d'une infinité transfinie d'ensembles mesurables (B) numerotés au moyen des nombres transfinies de seconde classe.

Et on voit sans peine que si un ensemble projectif de la seconde classe est donné effectivement, la décomposition en ensembles mesurables $(B)$ est donnée aussi effectivement.

En autre part, M. N. Lusin a énoncé dans sa note $\left({ }^{19}\right)$ une proposition suivante.

"La somme d'une infinité transfinie d'ensembles mesurables $(B)$ numérotés an moyen des nombres transfinis de seconde classe est un ensemble projectif de classe 2. D'ailleurs, si ce numérotage est effectif, la somme est un ensemble projectif de type $B_{2}{ }^{\text {"'. }}$.

Il me parait qu'il y a quelque contradiction entre la proposition de M. N. Lusin et le théorème 2 .

15. L'image continue uniforme d'un complémentaire analytique. Grâce au théorème 1 , on voit que tout ensemble projectif de la seconde classe peut être donné comme une image continue uniforme d'un complémentaire analytique. Or, concernant ce resultat, on peut établir un théorème suivant.

Théorème 3. Une image continue uniforme $E$ d'un complémentaire analytique $C$ est aussi un complémentaire analytique, si deux complémentaires analytiques $C_{1}$ et $C_{2}$ contenus dans $C$ sont transformés toujours en deux ensembles séparés au moyen des complémentaires analytiques.

Démonstration. Pour démontrer le théorème, il suffit de considérer le cas où les deux ensembles $C$ et $E$ sont linéaires en même temps. Or,

(18) N. Lusin, loc. cit., (13), 188.

(19) N. Lusin, Sur les ensembles analytiques nuls, Fund. Math., 25 (1935), 109-131. 
comme on peut choisir dans $R(y)$ un complémentaire analytique tel que $C$ est une image continue uniforme de cet ensemble, on peut supposer sans perdre la généralité, que $C$ est un sous-ensemble de $R(y)$. Soit $g(t)$ la transformation continue uniforme qui transforme $C$ en $E$. D'après le théorème de M. M. LAVRENTIÉFF $\left({ }^{20}\right)$, on peut prolonger la transformation $g(t)$ sur un ensemble $G \delta D$ de manière que $g(t)$ sont continue sur $D$ et qu'on ait $C<D<R(y)$. Or, d'après le théorème de M. S. MazurkiEwicz, l'ensemble $D$ est homéomorphe à un sous-ensemble fermé $N$ relativement à $R(y)$. On peut dire donc de plus, sans perdre la généralité, que le complémentaire analytique $C$ soit contenu dans $N$, et que $g(t)$ soit défini sur $N$. Or, $N$ est fermé relativement à $R(y)$, on peut prolonger $g(t)$ sur $R(y)$, de façons que $g(t)$ est continue sur $R(x)$. Considérons maintenant dans le plan $X O Y$ l'ensemble $K$ de tous les points $(g(t), t)$, où $t$ désigne un point de $C$. $K$ est alors un complémentaire analytique et la projection sur l'axe $O X$ de cet ensemble est $E$. Étant donné un intervalle $\left(n_{1}, n_{2}, \ldots n_{k}\right)$ de BAIRE sur $R(y)$, posons maintenant

$$
H_{n_{1} n_{2} \ldots n_{k}}=g\left(\left(n_{1} n_{2} \ldots n_{k}\right) C\right) .
$$

Soient $\left(n_{1}, n_{2}, \ldots n_{k}\right)$ et $\left(m_{1}, m_{2}, \ldots m_{k}\right)$ deux intervalles distincts de BAIRE d'ordre $k$. Comme les deux ensembles $\left(n_{1}, n_{2}, \ldots n_{k}\right) C$ et $\left(m_{1}, m_{2}, \ldots, m_{k}\right) \quad C$ sont les complémentaires analytiques disjoints et séparables au moyen des complémentaires analytiques, les deux ensembles $H n_{1} n_{2} \ldots n_{k}$ et $H m_{1} m_{2} \ldots m_{k}$ sont aussi séparables au moyen des complémentaires analytiques. On peut définir donc les complémentaires analytiques $J n_{1} n_{2} \ldots n_{k}$ tels qu'on ait

(1) $H n_{1} n_{2} \ldots n_{k}<J n_{1} n_{2} \ldots n_{k}$,

(2) pour deux intervalles distincts $\left(n_{1}, n_{2}, \ldots n_{k}\right)$ et $\left(m_{1}, m_{2}, \ldots, m_{k}\right)$ de BAIRE d'ordre $k$, nous avons $J_{n_{1} n_{2} \ldots n_{k}} J_{m_{1} m_{2} \ldots m_{k}}=0$,

Ici, on peut supposer, sans perdre la généralité, qu'on ait

(3) $J_{n_{1} n_{2} \ldots n_{k}<\overline{H n_{1} n_{2} \ldots n_{k}}}$, où $\overline{H n_{1} n_{2} \ldots n_{k}}$ désigne la ferméture de $H n_{1} n_{3} \ldots n_{k}$.

(4) $J_{n_{1} n_{2} \ldots n_{k}}>J_{n_{1} n_{2} \ldots n_{k} n_{k+1}}\left(k, n_{k}=1,2, \ldots\right)$.

En effet, il suffit de considérer au lieu de $J_{n_{1}} n_{2} \ldots n_{k}$ les ensembles $\overline{H n_{1} n_{2} \ldots n_{k}} \prod_{i=1}^{k} J n_{1} n_{2} \ldots n_{i}$. Or, puisque la transformation $g(t)$ est continue

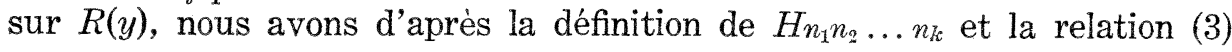

$$
\lim _{k \rightarrow \infty} \delta\left(H_{n_{1} n_{2} \ldots n_{k}}\right)=0,
$$

où $\delta\left(H n_{1} n_{2} \ldots n_{k}\right)$ désigne le diamètre de $H n_{1} n_{2} \ldots n_{k}$. Posons maintenant

(20 Voir, p. ex., C. Kuratowski, Topologie, I, (1933), Warszawa, p. 210. 
et

$$
J=\prod_{k=1}^{\infty} \sum_{n_{1} n_{2} \ldots n_{k}} J_{n_{1} n_{2} \ldots n_{k}}
$$

$$
J_{n_{1} n_{2} \ldots n_{k}}^{*}=J J_{n_{1} n_{2} \ldots n_{k}} \text {. }
$$

Les ensembles $J$ et $J_{n_{1}}^{*} n_{2} \ldots n_{k}$ sont alors les complémentaires analytiques. Or, pour un nombre fixe $k, J_{n_{1} n_{2} \ldots n_{k}}^{*}$ sont disjoints deux-à-deux, et $J=\sum_{n_{1} n_{2} \ldots n_{k}} J_{n_{1} n_{2} \ldots n_{k}}^{*} \cdot$ Par suite, l'ensemble $J_{n_{1} n_{2} \ldots n_{k}}^{*}$ est analytique relativement à l'ensemble $J$, d'où on peut choisir un ensemble analytique $M_{n_{1} n_{2} \ldots n_{k}}$ tel qu'on ait

$$
J_{n_{1} n_{2} \ldots n_{k}}^{*}=J M_{n_{1} n_{2} \ldots n_{k}} .
$$

Maintenant, posons

et

$$
Q=\prod_{k=1}^{\infty} \sum_{n_{1} n_{2} \ldots n_{k}} J_{n_{1} n_{2} \ldots n_{k}}^{*} \times\left(n_{1}, n_{2}, \ldots n_{k}\right)
$$

$$
M=\prod_{k=1}^{\infty} \sum_{n_{1} n_{2} \ldots n_{k}} M_{n_{1} n_{2} \ldots n_{k}} \times\left(n_{1}, n_{2}, \ldots n_{k}\right)
$$

Nous avons alors $Q=\{J \times R(y)\} M$. Or, on peut voir les propositions suivantes.

$1^{\circ}$, La projection sur l'axe $O X$ de l'ensemble $Q$ est l'ensemble $J$.

$2^{\circ}$, Quelque soit le point $x_{0}$ de $J$, l'ensemble $Q^{\left(x_{0}\right)}$ ne contient que un point.

En effet, supposons par impossible que $Q^{\left(x_{0}\right)}$ contient les deux points distincts $\left(x_{0}, y\right)$ et $\left(x_{0}, z\right)$. On peut alors choisir les deux intervalles distincts $\left(n_{1}, n_{2}, \ldots n_{k}\right)$ et $\left(m_{1}, m_{2}, \ldots m_{k}\right)$ de BAIRE d'ordre $k$ tels qu'on ait $y \in\left(n_{1}, n_{2}, \ldots n_{k}\right)$ et $z \in\left(m_{1}, m_{2}, \ldots m_{k}\right)$. Nous avons alors, d'après la définition de $\left(x_{0}, y\right)$ et $\left(x_{0}, z\right), x_{0} \in J_{n_{1} n_{2} \ldots n_{k}}^{*}$ et $x_{0} \in J_{m_{1} m_{2} \ldots m_{k}}^{*}$, ce qui entraine une contradiction.

$3^{\circ}, \quad K<Q$.

En effet, prenons un point $\left(x_{0}, y_{0}\right)$ de $K$, où $y_{0}=\left(n_{1}, n_{2}, \ldots\right)$. Nous avons alors $x_{0} \in H_{n_{1} n_{2} \ldots n_{k}}<J n_{1} n_{2} \ldots n_{k}$, d'où $\left(x_{0}, y_{0}\right) \in J_{n_{1} n_{2} \ldots n_{k}}^{*} \times\left(n_{1}, n_{2}\right.$, $\left.\ldots n_{k}\right)$ et par suite $\left(x_{0}, y_{0}\right) \in Q$.

D'ailleurs, comme $M_{n_{1} n_{2} \ldots n_{k}}\left(k, n_{k}=1,2, \ldots\right)$ sont les ensembles analytiques, l'ensemble $M$, et par suite $M-K$, est analytique. Done la projection sur l'axe $O X$ de l'ensemble $M-K$ est aussi analytique et

$$
\operatorname{Proj}_{O x}(Q-K)=J \operatorname{Projox}_{o x}(M-K),
$$


d'où la projection de $Q-K$ sur l'axe $O X$ est analytique relativement à $J$. Or, d'après les propositions $2^{\circ}$ et $3^{\circ}$, nous avons $\operatorname{Projox}_{o x} \times \operatorname{Proj}_{o x}(Q-K)=0$ et $J=\operatorname{Proj}_{o x} K+\operatorname{Proj}_{o x}(Q-K)$, c'est-à-dire,

$$
E=\operatorname{Proj}_{o x} K=J-\operatorname{Proj}_{o x}(Q-K),
$$

d'où l'ensemble $E$ est un complémentaire analytique.

C. Q. F. D.

De même, comme nous avons fait précedemment, on peut démontrer le théorème suivant sur la projection uniforme d'un ensemble projectif de la classe $\left(B_{2}\right)$.

Théorème 4. Pour qu'une image continue uniforme d'un ensemble $E$ projectif de la classe $\left(B_{2}\right)$ est aussi de la classe $\left(B_{2}\right)$, il faut et il suffit que deux ensembles projectifs de la classe $\left(B_{2}\right)$ contenus dans E soient transformés en deux ensembles aussi séparés au moyen des ensembles projectifs de la classe $\left(B_{2}\right)$.

16. L'image continue uniforme de l'ensemble de M. H. LEBESGUE. Étant donné dans le plan $X O Y$ le crible binaire $\Gamma$ de M. H. LebesGue, $\left({ }^{21}\right)$ désignons par $L$ l'ensemble de tous les points irrationnels binairement $x$ de l'intervalle $[0,1]$ tels que $\Gamma^{(x)}$ soit bien ordonné suivant la direction positive de l'axe $O Y$, et nous appelons $L$ l'ensemble de M. H. LebesGue. J'ai démontré recemment que tout ensemble $E$ linéaire non dénombrable analytique peut être représenté comme une image continue uniforme de l'ensemble $L$ de M. H. LEBesGue, quand on néglige une infinité dénombrable de point de $E\left({ }^{22}\right)$. Or, il serait très interéssant de savoir la classe de tous les ensembles donnés comme des images continues uniformes de $L$. On peut résoudre ce problème comme il suit. Tout d'abord nous donnerons quelque définition. Nous dirons que un ensemble $E$ contient partout des ensembles parfaits losequ'il existe un sous-ensemble parfait de $E$ dans tous les voisinages de chaque point de $E$. Nous avons alors le

Théorème 5. Tout ensemble $E$ linéaire non dénombrable projectif de la seconde classe qui contient partout des sous-ensembles parfaits peut être représenté comme une image continue uniforme de l'ensemble $L$ de $M$. $H$. LEBESGUE, quand, on néglige une infinité dénombrable de points de $E$. Et, toute image continue uniforme de l'ensemle $L$ est projectif de la seconde classe et contient partout des sous-ensembles parfaits.

Démonstration. Soit $E$ un ensemble linéaire non dénombrable projectif de la seconde classe qui contient partout des sous-ensembles parfaits.

(21) N. Lusin, loc. cit., (13), 199.

(22) M. Kondô, Sur la représentation paramétrique régulière des ensembles analytiques, Fund. Math., 31 (1938), 29-46, désignons par R. P. cette note dans la suite. 
Comme $E$ contient partout des sous-ensembles parfaits, de même que nous avons fait dans la démonstration de lemme 5 de R. P., on peut définir une suite dénombrable $\left\{E_{n}\right\}(n=1,2, \ldots)$ des ensembles $F_{\sigma}$ disjoints deux-àdeux et condensés dans $E$. Posons maintenant

$$
E^{*}=E-\sum_{n=1}^{\infty} E_{n} \text {. }
$$

Puis que l'ensemble $E^{*}$ est aussi projectif de la seconde classe, on peut définir d'après le théorème 1 un complémentaire analytique $W_{0}$ dans le plan $X O Y$ tel que la projection uniforme de $W_{0}$ sur l'axe $O X$ soit précisement l'ensemble $E$.

En désignant par $\left\{r_{n}\right\}(n=1,2, \ldots)$ les nombres rationnels, posons

$$
W=W_{0}+\sum_{n=1}^{\infty} E_{n} \times\left(r_{n}\right) \text {. }
$$

L'ensemble $W$ est un complémentaire analytique et nous avons

$$
\operatorname{Proj}_{o x} W=\text { Projox }_{0}+\sum_{n=1}^{\infty} E_{n}=E^{*}+\sum_{n=1}^{\infty} E_{n}=E,
$$

et de plus cette projection est uniforme. Or, on voit que $W$ contient partout des sous-ensembles parfaits. En effet, soient $p_{0}=\left(x_{0}, y_{0}\right)$ un point de $W$ et $\epsilon$ un nombre positif. Il existe alors un nombre rationnel $r_{n_{0}}$ tel qu'on ait $\left|y_{0}-r_{n_{0}}\right|<\frac{1}{2} \epsilon$. Le point $x_{0}$ est contenu dans $E$ et par suite, $x_{0}$ est un point condensation de $E$, d'où il existe un sous-ensemble parfait $P$ de $E_{n_{0}}$ tel qu'on ait dis $\left(x_{0}, p\right)<\frac{1}{2} \epsilon$ pour tout point $p$ de $P$. Or, $W$ contient l'ensemble $E_{n_{0}} \times\left(r_{n_{0}}\right)$ et par suite $P \times\left(r_{n_{0}}\right)$; et pour tout point $\left(p, r_{n_{0}}\right)$ de $P \times\left(r_{n_{0}}\right)$, nous avons

$$
\operatorname{dis}\left(\left(x_{0}, y_{0}\right),\left(p, r n_{0}\right)\right) \leqq \operatorname{dis}\left(x_{0}, p\right)+\operatorname{dis}\left(y_{0}, r_{n_{0}}\right)<\frac{1}{2} \epsilon+\frac{1}{2} \epsilon=\epsilon,
$$

c'est-à-dire, il existe dans un voisinage $U\left(p_{0} ; \epsilon\right)$ de $p_{0}$ du diamètre $\epsilon$ un sous-ensemble parfait de $W$. Il en résulte que l'ensemble $E$ est donné comme une image continue uniforme d'un complémentaire analytique $W$ quand on néglige une infinité dénombrable de points de $E$ et que $W$ contient partout des sous-ensembles parfaits. Or, d'après la démonstration du théorème de R. P., l'ensemble $W$ et par suite $E$, est une image continue uniforme de l'ensemble de M. H. LEBEsGuE, quand on néglige une infinité dénombrable de points de $W$. et $E$.

Puis, nous avons démontrer qu'une image continue uniforme de l'ensemble $L$ de M. H. LEBESGUE est un ensemble projectif de la seconde classe qui contient partout des sous-ensembles parfaits. Or, l'ensemble $L$ contient 
partout des sous-ensemble parfaits. En effet, supposons que le crible binaire $\Gamma$ de M. H. Lebesgue qui définit $L$ soit défini en dépendant d'une suite $\left\{r_{n}\right\}(n=1,2, \ldots)$ de nombres rationnels. Prenons les nombres rationnels $r_{n_{k}}(k=1,2, \ldots)$ tels que l'ensemble de nombres rationnels $r_{n_{k}}(k=1,2, \ldots)$ soit bien ordonné suivant la grandeur des nombres rationnels. Considérons tou point binairement irrationnel

$$
t=2^{-m_{1}}+2^{-m_{2}}+\cdots+2^{-m_{k}}+\cdots \quad\left(m_{1}<m_{2}<\cdots\right),
$$

tel que l'ensemble $M$ de nombres naturels $m_{k}(k=1,2, \ldots)$ soit contenu dans l'ensemble de nombres naturels $n_{k}(k=1,2, \ldots)$. L'ensemble $H$ de ces points ainsi obtenus est mesurable $(B)$ et indénombrable, et donc $H$ contient un sous-ensemble parfait. Or, comme $H$ est un sous-ensemble de $L$, l'ensemble $L$ contient un sous-ensemble parfait. Or, d'après le lemme 1 de R. P., $L$ est homogéne, c'est-à-dire, quel que soit l'intervalle ouvert I, II est homéomorphe à $L$. Par suite, $L$ contient partout des sousensembles parfaits. Il en résulte que une image continue uniforme de l'ensemble de LEBESGUE contient partout des sous-ensembles parfaits, et de plus, puisque $L$ est un complémentaire analytique, une image continue uniforme de $L$ est d'après le théorème 1 un ensemble projectif de la seconde classe.

C. Q. F. D.

17. L'uniformisation des ensembles projectifs de la seconde classe. $E$ étant un ensemble projectif de la seconde classe contenu dans la plan $X O Y$. On peut alors choisir dans l'espace $X O Y Z$ un complémentaire analytique $C$ tel que la projection de $C$ sur le plan $X O Y$ soit précisement $E$. Etant donnée une transformation $y^{\prime}=\sigma(y, z)$ qui établit une homéomorphe généralisée $(0,2)$ au sens de M. C. KURATOwski entre la plan $Y O Z$ et l'axe $O Y$, considérons une transformation $\varphi(p): x^{\prime}=x, y^{\prime}=\sigma(y, z)$ qui transforme l'espace $X O Y Z$ en la plan $X O Y$. D'après la définition de la transformation $\varphi(p), \varphi(C)$ est aussi un complémentaire analytique dans le plan $X O Y$. On peut donc d'après le théorème de l'uniformisation trouver un complémentaire analytique $U$ qui est un uniformisateur de $\varphi(C)$. Or, comme nous avons $(\varphi(C))^{(x)}=\varphi\left(C^{(x)}\right)$ pour tout point de l'axe $O X$, l'ensemble $U^{(x)}$ et par suite $\left(\varphi^{-1}(U)\right)^{(x)}$ contient un point au plus pour tout point $x$ l'axe $O X$, et de plus $\left(\varphi^{-1}(U)\right)^{(x)}$ est vide ou non suivant que $C^{(x)}$ est vide ou non. Par conséquent, la projection de $\phi^{-1}(U)$ sur le plan $X O Y$ est un uniformisateur de $E$. Or, $\phi^{-1}(U)$ est d'après la définition de $\varphi(p)$ un complémentaire analytique, et donc la projection de $\phi^{-1}(U)$ sur le plan $X O Y$ est projectif de la seconde classe. Nous avons donc le

Théorème 6. Tout ensemble plan projectif de la seconde classe peut être uniformisé au moyen des ensembles de la même classe. 
Puis, nous allons considérer l'uniformisation des ensembles projectif de la classe $\left(B_{2}\right) . \quad E$ étant un ensemble plan projectif de la classe $\left(B_{2}\right)$; comme nous avons fait plus haut, on peut choisir dans l'espace $X O Y Z$ un complémentaire analytique $C$ tel que la projection uniforme de $C$ sur le plan $X O Y$ soit $E$, et un complémentaire analytique $U$ contenu dans $C$ tel que pour tout point $x$ de la projection de $E$ sur l'axe $O X U^{(x)}$ soit précisement un point. Il est alors évident que la projection de $U$ sur le plan $X O Y$ est une uniformisation de $E$. Nous avons, d'après la définition de $C$, $\operatorname{Proj}_{X O Y}(C-U)+\operatorname{Proj}_{X O Y} U=E$ et $\operatorname{Proj}_{X O Y}(C-U) \times \operatorname{Proj}_{X O Y} U=0$, d'où les deux ensembles $\operatorname{Proj}_{X O Y}(C-U)$ et $\operatorname{Proj}_{X O Y} U$ sont projectifs de la seconde classe. Or, comme $E$ est projectif de la classe $\left(B_{2}\right), \operatorname{Proj}_{X O Y} U$ est aussi projectif de la classe $\left(B_{2}\right)$. Par conséquent nous avons le

Théorème 7. Tout ensemble projectif de la classe $\left(B_{2}\right)$ peut être uniformisé au moyen des ensembles de la même classe.

Remarque. M. A. LiapounofF a démonté récemment le théorème suivant dans sa note "Sur l'uniformisation des complémentaires analytiques", (Recueil Mathématique, N. S., t. 3 (1938), p. 219-223).

Tout complémentaire analytique plan peut être uniformisé au moyen d'un ensemble $C A_{2}$.

Or, ce resultat est contenu dans le théorème de l'uniformisation comme le cas particulier. 